\title{
Demarcating circulation regimes of synchronously rotating terrestrial planets within the habitable zone
}

\author{
Jacob Haqq-Misra ${ }^{1,2}$, Eric. T. Wolf ${ }^{3}$, Manoj Joshi ${ }^{4,5}$, Xi Zhang ${ }^{6}$, and Ravi Kumar \\ $\mathrm{Kopparapu}^{1,2,7,8}$
}

\begin{abstract}
We investigate the atmospheric dynamics of terrestrial planets in synchronous rotation within the habitable zone of low-mass stars using the Community Atmosphere Model (CAM). The surface temperature contrast between day and night hemispheres decreases with an increase in incident stellar flux, which is opposite the trend seen on gas giants. We define three dynamical regimes in terms of the equatorial Rossby deformation radius and the Rhines length. The slow rotation regime has a mean zonal circulation that spans from day to night side, with both the Rossby deformation radius and the Rhines length exceeding planetary radius, which occurs for planets around stars with effective temperatures of $3300 \mathrm{~K}$ to $4500 \mathrm{~K}$ (rotation period $>20$ days). Rapid rotators have a mean zonal circulation that partially spans a hemisphere and with banded cloud formation beneath the substellar point, with the Rossby deformation radius is less than planetary radius, which occurs for planets orbiting stars with effective temperatures of less than $3000 \mathrm{~K}$ (rotation period $<5$ days). In between is the Rhines rotation regime, which retains a thermally-direct circulation from day to night side but also features midlatitude turbulence-driven zonal jets. Rhines rotators occur for planets around stars in the range of $3000 \mathrm{~K}$ to $3300 \mathrm{~K}$ (rotation period $\sim 5$ to 20 days), where the Rhines length is greater than planetary radius but the Rossby deformation radius is less than planetary radius. The dynamical state can be observationally inferred from comparing the morphology of the thermal emission phase curves of synchronously rotating planets.
\end{abstract}

\footnotetext{
${ }^{1}$ Blue Marble Space Institute of Science, 1001 4th Ave Suite 3201, Seattle, WA 98154, USA

${ }^{2}$ NASA Astrobiology Institute's Virtual Planetary Laboratory, P.O. Box 351580, Seattle, WA 98195, USA

${ }^{3}$ Laboratory for Atmospheric and Space Physics, Department of Atmospheric and Oceanic Sciences, University of Colorado, Boulder, Colorado, USA

${ }^{4}$ School of Environmental Sciences, University of East Anglia, Norwich Research Park, Norwich NR4 7TJ, UK

${ }^{5}$ Climatic Research Unit, University of East Anglia, Norwich Research Park, Norwich NR4 7TJ, UK

${ }^{6}$ Earth and Planetary Sciences Department, University of California, Santa Cruz, California, USA

${ }^{7}$ NASA Goddard Space Flight Center, 8800 Greenbelt Road, Mail Stop 699.0 Building 34, Greenbelt, MD 20771, USA

${ }^{8}$ Department of Astronomy, University of Maryland, College Park, MD 20742, USA
} 
Subject headings: planets and satellites: atmospheres, planets and satellites: terrestrial planets, stars: low-mass, astrobiology

\section{Introduction}

M-dwarf stars provide an abundance of environments for potentially hosting habitable planets. The discoveries of Proxima Centauri b around our closest stellar neighbor (Anglada-Escudé et al. 2017) and the seven planets of the TRAPPIST-1 system (Gillon et al. 2017) indicate that Mdwarfs can harbor terrestrial plants within their liquid water habitable zones (Kasting et al. 1993; Selsis et al. 2007; Kopparapu et al. 2013, 2014; Yang et al. 2013, 2014), which makes them likely candidates for upcoming surveys with JWST and TESS. Due to the small size of their host stars, and their short period orbits, habitable planets around M-dwarf stars are optimal targets for detection and characterization of their atmospheres.

Speculation that planets in orbit around low-mass stars would be prone to synchronous rotationso that one side experiences permanent day, while the other experiences permanent night - initially raised concern that such planets would be prone to freeze out their atmospheres and thus might not be habitable at all (Dole 1964). But subsequent investigations with simplified climate models (Haberle et al. 1996) and general circulation models (GCMs) (Joshi et al. 1997; Joshi 2003;

Merlis \& Scheider 2010; Edson et al. 2011; Showman et al. 2010, 2013; Leconte et al. 2013; Yang et al. 2013, 2014; Cullum et al. 2014; Hu \& Yang 2014; Carone et al. 2014, 2015, 2016; Wordsworth 2015; Way et al. 2016; Kopparapu et al. 2016, 2017; Noda et al. 2017; Fujii et al. 2017) have demonstrated that energy transport from the day to night hemisphere is generally sufficient to avoid atmospheric collapse across a wide range of atmospheric compositions and rotation periods.

Further analysis has revealed common patterns in the large-scale dynamics of synchronously rotating terrestrial planets, most notably a transition between circulation regimes as a planet's rotation period decreases and the Rossby deformation radius approaches planetary radius (Merlis \& Scheider 2010; Edson et al. 2011; Showman et al. 2010, 2013; Leconte et al. 2013; Yang et al. 2013, 2014; Haqq-Misra \& Kopparapu 2015; Carone et al. 2014, 2015, 2016; Noda et al. 2017). For an Earthsize synchronously rotating planet, this transition occurs at a rotation period of $\sim 5$ days (Edson et al. 2011; Carone et al. 2015). Recently, Noda et al. (2017) explored the dependence of large-scale dynamics on a wide range of rotation periods and identified four distinct dynamical regimes for synchronously rotating terrestrial planets, some of which are not explained by changes in the Rossby deformation radius alone.

In this paper we discuss the dynamical regimes that characterize the atmospheres of habitable moist terrestrial planets in synchronous rotation around M-dwarf stars. Using the simulations conducted by Kopparapu et al. (2017), we examine the temperature contrast between the day and night side as these planets move toward the inner edge of the habitable zone. We then define three distinct dynamical regimes based upon the equatorial Rossby deformation radius and the Rhines 
length, which define the most salient features of a planet's large-scale atmospheric dynamics. Such dynamical states could potentially be distinguished in future missions through observations of thermal emission phase curves.

\section{Model Description}

The set of GCM simulations by Kopparapu et al. (2017) represent Earth-sized terrestrial planets with 1-bar $\mathrm{N}_{2}$ atmospheres, where water vapor is the only greenhouse gas. Planets are assumed to be aquaplanets covered in a swamp ocean; thus, water is abundant in the system, limited only by the Clausius-Clapeyron relation. Calculations are performed at increasing stellar flux up to the inner edge of the habitable zone, where the model atmosphere becomes unstable with the initiation of a runaway greenhouse. Simulated planets have global mean surface temperatures ranging between $\sim 250-310 \mathrm{~K}$. This set of calculations is conducted with six different spectral energy distributions representing a range of M-dwarf host stars, with effective temperatures of $4500 \mathrm{~K}, 4000 \mathrm{~K}, 3700 \mathrm{~K}$, $3300 \mathrm{~K}, 3000 \mathrm{~K}$, and $2600 \mathrm{~K}$ using the BT-SETTL grid of models (Allard et al. 2007).

We assume all planets are in synchronous rotation with their host stars. This implies that the rotation period and orbital period must be equal, which we calculate self-consistently for each case using Kepler's third law (Wordsworth 2015; Kopparapu et al. 2016, 2017) as

$$
P=\left[\left(\frac{L_{\star}}{L_{\odot}}\right)\left(\frac{F_{\oplus}}{F_{\star}}\right)\right]^{\frac{3}{4}}\left[\frac{M_{\odot}}{M_{\star}}\right]^{\frac{1}{2}} .
$$

Here $P$ is the orbital (and rotational) period of the planet in years, $L_{\star} / L_{\odot}$ is the luminosity of the host star scaled by the luminosity of the sun, $F_{\star} / F_{\oplus}$ is the incident stellar flux on the planet scaled by the incident solar flux on Earth, and $M_{\star} / M_{\odot}$ is the mass of the host star in solar mass units.

These simulations were all conducted with a modified version of the Community Atmosphere Model (CAM) from the National Center for Atmospheric Research (NCAR) in Boulder, Colorado. This version of CAM includes updates to the native radiative transfer by implementing a new correlated- $k$ method based on the HITRAN 2012 database, as well as increases to the infrared spectral resolution. The radiative transfer in this version of CAM is valid for $\mathrm{N}_{2}-\mathrm{H}_{2} \mathrm{O}$ atmospheres with surface pressures up to 10 bar, and the GCM dynamical core includes the contribution of condensing water vapor to the surface pressure tendency. For a detailed discussion of this implementation of CAM, see Kopparapu et al. (2017).

\section{Day-Night Surface Temperature Contrast}

In order to detail the effectiveness of day-night energy transport across our simulation set, we examine the surface temperature difference between the day side and night side hemispheres. Let $T_{d a y}$ be the area-weighted surface temperature over the day side (substellar) hemisphere and $T_{\text {night }}$ 
be the area-weighted surface temperature over the night side (antistellar) hemisphere. We plot the day-night temperature difference in the left panel of Fig. 1 versus relative stellar flux, which shows that the day-night temperature difference decreases as a planet warms. Fig. 1 includes markers at each of the GCM simulations conducted, labels for each stellar spectral type (left panel), labels for rotation period (right panel), and stellar flux scaled relative to the present-day solar flux, $S_{0}$. These simulations represent climate states ranging from the middle to inner edge of the habitable zone for 1-bar $\mathrm{N}_{2}-\mathrm{H}_{2} \mathrm{O}$ atmospheres in synchronous rotation.

Previous studies using gray (i.e., non-wavelength-dependent) radiative transfer have suggested that the day-night temperature difference shows no dependence upon rotation period for cloud-free atmospheres (Merlis \& Scheider 2010; Noda et al. 2017). Other studies have demonstrated that the presence and pattern of clouds depends on rotation rate, which alters the pattern of incident stellar radiation absorbed at the surface (Yang et al. 2014; Kopparapu et al. 2016, 2017). Such experiments that separate the effects of incident stellar flux, stellar spectrum, and rotation rate are instructive for improving theoretical understanding of planetary atmospheres; however, Eq. (1) states that stellar flux and rotation period are inseparably linked for any synchronously rotating planets that are observed. Our goal with this paper is an attempt to apply the knowledge gained from these previous theoretical investigations toward the astronomically self-consistent set of GCM simulations by Kopparapu et al. (2017).

The left panel of Fig. 1 1 shows that at a fixed value of relative stellar flux, planets around hotter stars have a larger value of day-night temperature difference. This increased day-night temperature difference occurs due to changes in both rotation period and the spectral energy distribution. These two effects are difficult to separate and can only be accounted for in a GCM with non-gray (i.e. wavelength-dependent) radiative transfer. Although rotation period by itself is insufficient to explain the changes in day-night temperature difference, it remains a contributing factor when comparing synchronously rotating terrestrial planets around stars with different effective temperatures.

The right panel of Fig. 1 shows that equilibrium temperature, $T_{e q}$, increases as relative stellar flux increases, where $T_{e q}^{4}=S(1-\alpha) / 4 \sigma$ (where $S$ is stellar flux, $\alpha$ is top-of-atmosphere albedo, and $\sigma$ is the Stefan-Boltzman constant). Equilibrium temperature is a measure of the energy balance of planet, with the contributions of dynamical and physical processes to radiation balance (such as clouds and surface albedo) encapsulated in the planetary albedo parameter, $\alpha$. We note that hotter stars have a lower equilibrium temperature than lower stars (when $S / S_{0}$ is constant), which occurs because cooler stars emit a lower percentage of visible radiation that can be absorbed at the planet's surface. In our discussion that follows, we scale our temperature differences by $T_{e q}$ in order to show the relative contribution of direct stellar warming compared to atmospheric warming.

We define the day-night temperature contrast as $\left(T_{d a y}-T_{\text {night }}\right) / T_{e q}$ (following the same approach as Koll \& Abbot (2016)), which provides a non-dimensional parameter for comparing simulations with different host stars. We plot this day-night temperature contrast in the top left panel 
of Fig. 2 as a function of relative stellar flux, where higher values represent greater day-night contrast. The day-night temperature contrast (top left panel of Fig. 21) decreases as relative stellar flux increases, which shows similar functional morphology as the day-night temperature difference (left panel of Fig. 1).

We next consider how the mean surface temperature in our simulations compares with the daynight temperature contrast. The top right panel of Fig. 2 shows that all planets in our simulation set exhibit a decrease in the day-night temperature contrast as the global mean surface temperature increases. All cases in the simulation set show a strong correlation between day-night temperature contrast and mean surface temperature, with the warmest simulations showing the smallest daynight temperature contrast. (Here and elsewhere, we reverse the horizontal axis so that the warmest simulations are consistently at the left side of the panel.) The decrease in day-night temperature contrast correlates with a rise in total greenhouse effect as the stellar flux increases, where total greenhouse effect, $F_{G H}$, is calculated as the difference between the surface outgoing longwave flux and top-of-atmosphere infrared flux, $F_{O L R}$, so that

$$
F_{G H}=\sigma T_{s}^{4}-F_{O L R}
$$

Eq. (2) suggests two possibilities for causing a change in the greenhouse effect as stellar flux increases. Dry energy transport that yields net warming provides one mechanism that can increase $T_{s}$ and thus increase $F_{G H}$. Moist processes provide a second mechanism, with the accumulation of water vapor (the only greenhouse gas in our simulations) causing a decrease in $F_{O L R}$ that likewise increases $F_{G H}$. The bottom left panel of Fig. 2 shows that the difference in greenhouse effect between the day side and the night side decreases as the planet warms. That is, as stellar flux increases for these planets, the magnitude of greenhouse effect approaches equality between day and night hemispheres.

The dry contribution to the decrease in day-night temperature contrast can be explained by an increase in the static energy flux convergence on the night side. Static energy, $s$, is the sum of an air parcel's internal energy, $c_{p} T$, (i.e., enthalpy), potential energy due to gravity, $\Phi$, and latent energy due to moisture, $L_{v} q$. (Here $c_{p}$ is the heat capacity of air at constant pressure, $\Phi$ is geopotential, $L_{v}$ is the enthalpy of vaporization of water, and $q$ is specific humidity.) The static energy flux, $\mathbf{v} s$, represents the change in static energy due to wind (where $\mathbf{v}$ is the horizontal wind vector), while the static energy flux convergence, $-\nabla \cdot(\mathbf{v} s)$, describes the inward flow of static energy. An increase in static energy flux convergence on the night side will lead to an increase in internal energy, which, by Eq. (2), causes $F_{G H}$ to increase. Ultimately, this increase in static energy flux convergence occurs as a response to the deepening pressure contrast between day and night hemispheres as a synchronously rotating planet warms, which increases divergence on the day side and convergence on the night side of the component of wind known as the isallobaric wind (see Appendix $\mathrm{A}$ for additional discussion). As these planets warm due to an increase in stellar flux, this increase in static energy flux divergence on the day side and convergence on the night side causes the temperature difference between the two hemispheres to decrease. 
The bottom right panel of Fig. 2 shows the vertically-integrated static energy flux convergence per unit mass on the night hemisphere. This quantity increases as the day-night temperature contrast decreases, which corresponds to a similar increase in vertically-integrated static energy flux divergence on the day hemisphere (not shown). Solid lines in this figure show the flux convergence with only the dry components of static energy $\left(c_{p} T+\Phi\right)$, while dashed lines indicate the static energy flux convergence with moisture included $\left(c_{p} T+\Phi+L_{v} q\right)$. The dry static energy dominates the flux convergence, with minimal effects of latent heating evident only in the warmest simulations. For most simulations, the moist (dashed) static energy flux convergence cannot be distinguished from the dry (solid) static energy flux convergence, which reveals that the contribution of the static energy flux convergence to the day-night temperature contrast is an inherently dry phenomenon that does not necessarily depend upon latent heating from moisture.

Many of our simulations also feature a night side temperature inversion above the surface (up to about $800 \mathrm{hPa}$ ). Such inversion layers also appear in other GCM simulations of synchronously

rotating atmospheres (Joshi et al. 1997; Merlis \& Scheider 2010; Leconte et al. 2013) and can be replicated in simpler radiative-convective subsiding models (Koll \& Abbot 2016). Note, similar temperature inversions are observed in the cold, dry, and dark polar winters on Earth (Curry et al. 1996; Liu et al. 2006), which provide an analog to the dark antistellar hemispheres of synchronously rotating worlds. In such cases the atmosphere radiates from the layer above this inversion, which provides additional infrared flux into the surface and can even contribute to a negative net greenhouse effect, $F_{G H}<0$, for some of the coldest simulations. The inversions, both for antistellar hemispheres and for polar winters on Earth, are maintained by vigorous radiative cooling of land and ice surfaces along with the transport of overlying warmer air masses in the free atmosphere (Bintanja et al. 2011).

Unlike Earth's poles, the antistellar hemispheres of synchronously rotating planets always remains dark, and thus the inversion can be a permanent feature. However, the night side inversion decreases in strength as the planet warms, with the destruction of the inversion triggered by the increase in dry static energy flux convergence on the night side near the surface (Fig. 2). Warming of the night side surface layers from dry static energy convergence then increases water vapor abundance, which increases the night side water vapor greenhouse effect and causes further warming. Here, we find that the night side inversions vanish for planets with mean surface temperatures of $\geq 300 \mathrm{~K}$. Water vapor increases in both hemispheres as stellar flux increases and the surface warms. While the absolute magnitude of greenhouse effect increases all across the planet as the atmosphere warms, the difference in the greenhouse effect between day and night side lessens, which contributes toward reducing the day-night temperature contrast.

\subsection{Comparison with Gray Analytic Theory}

The contributions from water vapor accumulation and dry energy transport to total greenhouse warming in Eq. (2) can be represented as an equivalent optical gray depth, which summarizes this 
combined warming in a parameter that can be compared with gray analytic theory. Koll \& Abbot (2016), following a similar approach as Wordsworth (2015), analyze the atmospheres of dry synchronously rotating planets by drawing upon Carnot's theorem to describe the scaling of surface winds as a heat engine. Using a gray analytic two-column model, Koll \& Abbot (2016) develop expressions for $T_{d a y}$ and $T_{\text {night }}$,

$$
T_{d a y} \approx 2^{1 / 4} T_{e q}\left[1-\frac{3 \tau_{L W}}{4\left(1+4 \frac{R}{c_{p}}\right)}\right]
$$

and

$$
T_{\text {night }} \approx 2^{1 / 4} T_{e q} \frac{\tau_{L W}^{1 / 4}}{\left(1+4 \frac{R}{c_{p}}\right)^{1 / 4}},
$$

where $\tau_{L W}$ is the globally-averaged longwave optical depth and $R$ is the dry gas constant. Eq. (3) shows that the day side temperature decreases with $\tau_{L W}$, while Eq. (4) reveals that the night side temperature increases with $\tau_{L W}$. Combining Eqs. (3) and (4) to obtain $\left(T_{\text {day }}-T_{\text {night }}\right) / T_{e q}$ shows that the day-night temperature contrast decreases as $\tau_{L W}$ increases. This remains consistent with our model results that show an increase in greenhouse effect, analogous to an increase in $\tau_{L W}$, associated with the decrease in day-night temperature contrast.

Substituting values of $\left(T_{\text {day }}-T_{\text {night }}\right) / T_{\text {eq }}$ from our results into Eqs. (3) and (4) allows us to calculate an equivalent gray optical depth for our set of GCM calculations, which we show in the left panel of Fig. 3. In our case, the parameter $\tau_{L W}$ represents any process that contributes to greenhouse warming (Eq. (2)), which includes both the accumulation of water vapor and increase in static energy flux convergence as stellar flux increases, as well as cloud processes. Although the analytic model of Koll \& Abbot (2016) focuses on dry atmospheres, this relationship between $\tau_{L W}$ and $F_{G H}$ still remains qualitatively consistent with our non-gray, moist GCM results.

The Koll \& Abbot (2016) heat engine analogy continues by predicting that mean day side surface wind, $U_{s}$, should scale as $U_{s}^{3} \sim\left(T_{d a y}-T_{e q}\right)\left(1-e^{-\tau_{L W}}\right) T_{e q}^{4}$. This expression, and a similar one developed by Wordsworth (2015), compares favorably with dry GCM calculations that use gray radiative transfer; however, applying this relationship to our moist GCM results by substituting from Eqs. (3) and (44) suggests that $U_{s}$ should increase as the planet warms. However, Fig. 3 (middle panel) shows $U_{s}$ calculated from our GCM simulations as the area-weighted root mean squared surface wind on the day hemisphere, which shows that the simulations with the smallest day-night temperature contrast have the smallest value of mean day side wind. Fig. 2 (upper left) shows that these simulations with the smallest day-night temperature contrast also show the warmest mean surface temperatures. This implies that $U_{s}$ in our simulations tends to decrease as a planet moves toward the inner edge of the habitable zone and day-night temperature contrast shrinks. Likewise, the strength of the zonal overturning (i.e., Walker) circulation also tends to decrease as the planet moves toward the inner edge of the habitable zone (Fig. 3, right panel). These results from our moist GCM simulations are somewhat inconsistent with dry analytic theory, 
by showing a decrease in wind speed and zonal circulation strength due to the changes in both stellar insolation and planetary rotation period.

The theoretical expression of Koll \& Abbot (2016) remains valid when we examine our results at a fixed value of day-night temperature contrast. Fig. 3 shows that at a fixed value of day-night temperature contrast (e.g., $\left.\left(T_{d a y}-T_{\text {night }}\right) / T_{e q}=0.12\right)$, the mean day side surface wind increases with stellar effective temperature (middle panel) as does the maximum Walker circulation strength (right panel). Stars with a higher stellar effective temperature emit a higher proportion of energy at shorter wavelengths, which corresponds to additional surface heating on planets orbiting such stars.

Water vapor absorption is one key feature present in our GCM that is absent in the gray analytic model of Koll \& Abbot (2016). Stars with a lower stellar effective temperature have stronger emission at infrared wavelengths, which allows for greater absorption of this incoming radiation by water vapor in the atmosphere. This effect is evident in Fig. 3; planets orbiting the hottest stars are largely transparent to incoming stellar radiation, which causes greater surface warming and leads to the strongest circulation and surface winds. Likewise, planets orbiting cooler stars absorb a larger fraction of incoming radiation, which causes less direct surface warming and leads to a reduction in the maximum Walker circulation strength.

In general, the analytic expressions by Koll \& Abbot (2016) can adequately describe variations in $U_{s}$ for large changes in $\tau_{L W}$ (such as comparing planets with fixed day-night temperature contrast around stars of different spectral type), but this relationship breaks down when considering the smaller changes in surface pressure and wind that occurs as a terrestrial planet is moved closer toward the inner edge of the habitable zone.

\subsection{Comparison with Gas Giants}

The day-night temperature contrast is one of the primary observable features of exoplanet atmospheres. The decreasing trend of day-night surface temperature contrast with increasing equilibrium temperature from our terrestrial simulations is opposite for synchronously rotating gas giant planets, where observations show that an increase in heating leads to an increase in the day-night temperature contrast (Perez-Becker \& Showman 2013; Komacek \& Showman 2016; Komacek et al. 2017). The day-night temperature contrast observed on an optically thick gas giant atmosphere occurs at the emission level in the free atmosphere, whereas the flux emitted by an optically thin terrestrial atmosphere primarily emerges from the surface. These opposite trends imply that the two types of atmospheres are located in two fundamentally different regimes: a hot, dry, top-heated gas giant regime versus a cold, moist, bottom-heated terrestrial regime.

For both of these regimes, the day-night heat transport in the free atmosphere of synchronously rotating planets is strongly influenced by radiation and zonally propagating waves (Showman et al. 2013; Perez-Becker \& Showman 2013; Wordsworth 2015; Koll \& Abbot 2015, 2016; Komacek \& Showman 
2016; Komacek et al. 2017; Zhang \& Showman 2017). The free-atmosphere day-night temperature contrast can be predicted based on a scaling theory developed by Komacek \& Showman (2016); Komacek et al. (2017) and Zhang \& Showman (2017),

$$
\left.\frac{T_{\text {day }}-T_{\text {night }}}{T_{e q}}\right|_{\text {free }} \sim 1-\frac{2}{\alpha+\sqrt{\alpha^{2}+4 \gamma^{2}}},
$$

where the non-dimensional parameters $\alpha$ and $\gamma$ are defined as:

$$
\begin{aligned}
& \alpha=1+\frac{\left(\Omega+\frac{1}{\tau_{\text {drag }}}\right) \tau_{\text {wave }}^{2}}{\tau_{\text {rad }} \Delta \ln p}, \\
& \gamma=\frac{\tau_{\text {wave }}^{2}}{\tau_{\text {rad }} \tau_{\text {adv }, e q} \Delta \ln p} .
\end{aligned}
$$

Here $\Omega$ is rotation rate, $\tau_{\text {rad }}$ is the radiative timescale, $\tau_{\text {wave }}$ is the timescale for wave propagation, $\tau_{d r a g}$ is the frictional drag timescale, $\Delta \ln p$ is the thickness of the photosphere in terms of $\log$ pressure, and $\tau_{a d v \text { ea }}$ is the advective timescale due to the "equilibrium cyclostrophic wind". (See Appendix A from Zhang \& Showman (2017) for more discussion.)

The key parameters here are the radiative timescale, $\tau_{\text {rad }}$, and wave propagation timescale, $\tau_{\text {wave }}$. The typical radiative timescale on a canonical hot Jupiter is about $10^{4}-10^{6} \mathrm{~s}$ in the photosphere (Showman \& Guillot 2002). The gravity wave speed, $(g H)^{1 / 2}$ where $g$ is gravitational acceleration and $H$ is scale height, is on the order of $1 \mathrm{~km} \mathrm{~s}^{-1}$, resulting a wave propagation timescale of $10^{4}-10^{5} \mathrm{~s}$, which is comparable to the radiative timescale. However, the temperature dependence of the two timescales is different. In the dry atmosphere of a gas giant, $\tau_{\text {rad }} \propto T_{e q}^{-3}$ (Showman \& Guillot 2002) if opacity does not significantly change with temperature. On the other hand, in an isotherm limit, $\tau_{\text {wave }} \propto T_{e q}^{-1}$. As equilibrium temperature increases, the radiative timescale decreases more rapidly than the wave propagation timescale. Eq. (51) predicts that the day-night temperature contrast will increase. Physically, the atmosphere of a gas giant will become more radiation-controlled and the day-night heat transport will be less efficient by waves, which results in a larger day-night temperature contrast. Simulations by Komacek \& Showman (2016); Komacek et al. (2017) have confirmed this trend in the hot atmosphere regime for giant planets.

By contrast, due to their colder temperature and smaller planetary radius, terrestrial atmospheres in the habitable zone generally show a much shorter wave propagation timescale compared to the radiative timescale (Selsis et al. 2011; Koll \& Abbot 2015). For example, a typical radiative timescale on an Earth-like planet is a few days, but the gravity wave propagation timescale is about an hour due to its smaller planetary radius. Thus the ratio $\tau_{\text {wave }} / \tau_{\text {rad }}$ is on the order of 0.01 or less (Koll \& Abbot 2015). This implies that the temperature of the day side and night side are homogenized in the free atmosphere, which leads to the "weak temperature gradient" regime for terrestrial atmospheres (Wordsworth 2015; Koll \& Abbot 2015, 2016). Unlike the gas giant regime, which are primarily heated from the top, the atmospheres of optically thin terrestrial planets are primarily heated from the bottom. Surface temperatures, both on the day and night side, are 
approximately in radiative-convective equilibrium with the overlying atmospheres (Koll \& Abbot 2016). As a result, the day-night surface temperature contrast in the terrestrial regime is primarily governed by the opacity that controls the surface-atmosphere radiative flux exchange, as shown by Eqs. (3) and (4). Given a particular stellar flux, increasing the opacity will tend to decrease the day side surface temperature but increase the night side surface temperature (Koll \& Abbot 2016), which therefore decreases the day-night temperature contrast at surface. If opacity does not change significantly with equilibrium temperature in the dry atmosphere, the day-night temperature contrast will be approximately constant for all planets. In thin moist atmospheres, the presence of water vapor, and its associated increase in longwave opacity with equilibrium temperature, leads to a decreasing trend of day-night surface temperature contrast.

\section{Dynamical Regimes}

We analyze three regimes for large-scale atmospheric dynamics on synchronously rotating planets - slow rotators, rapid rotators, and Rhines rotators - and their dependence on the Rossby deformation radius and the Rhines length. We define and discuss the relevant dynamical parameters for each of these cases below, which we compare with previous GCM studies that have examined the dynamical regimes of synchronously rotating planets. We show that terrestrial planets in the habitable zone of stars with an effective stellar temperature of $3700 \mathrm{~K}$ to $4500 \mathrm{~K}$ are in the slow rotation regime, while those around $2600 \mathrm{~K}$ stars are in the rapid rotation regime. Intermediate stellar cases in the range of $3000 \mathrm{~K}$ to $3300 \mathrm{~K}$ show a unique transitional dynamical state that we describe as the Rhines rotation regime.

These three dynamical regimes are defined using two parameters: the Rossby deformation radius, which constrains the maximum extent of the zonal overturning circulation, and the Rhines length, which determines the maximum extent of zonally-elongated turbulent structures. We summarize this approach for characterizing the atmospheric dynamics of a synchronously rotating planet in Fig. 4. Slow rotators are found in the upper-right quadrant of Fig. 4, where the nondimensional Rossby deformation radius and non-dimensional Rhines length are both greater than one. Rapid rotators occur in the lower-left quadrant of Fig. 4, where both the non-dimensional Rossby deformation radius and non-dimensional Rhines length are less than one. Rhines rotators describe a transitional dynamical state, which occurs in the lower-right quadrant of Fig. 4 with a non-dimensional Rossby deformation radius greater than one but a non-dimensional Rhines length less than one. We apply these regimes below in our discussion of terrestrial planets near the inner edge of the habitable zone, but this classification scheme can be generalized to planets with less Earth-like atmospheres, including synchronously rotating giant planets. 


\subsection{Slow Rotators}

Slow rotators are characterized by strong convective motion beneath the substellar point, with energy transport by Rossby and Kelvin waves from the day to night side of the planet. Atmospheric dynamics on a slow rotator are broadly characterized by a thermally-direct circulation with heating and rising air on the day side, cooling and descending air on the night side, and the strength of the circulation limited by frictional dissipation in the boundary layer (Koll \& Abbot 2016). Slow rotators are equivalent to the 'Type-I' circulation regime described by Noda et al. (2017), where the thermally-direct day-night circulation is the primary characteristic of the planet's large-scale dynamics.

Previous studies have demonstrated that maintaining this hemispheric large-scale circulation requires that the Rossby deformation radius is greater than the planetary radius (Merlis \& Scheider 2010; Edson et al. 2011; Showman et al. 2010, 2013; Leconte et al. 2013; Yang et al. 2014; Haqq-Misra \& Kopparap 2015; Carone et al. 2014, 2015, 2016; Noda et al. 2017). The Rossby deformation radius represents the ratio of buoyancy to rotational forces. For synchronously rotating planets, the Rossby deformation radius is proportional to the maximum extent of the mean zonal circulation from day to night side. Because we are primarily concerned with the equatorial propagation of Rossby, Kelvin, and other waves, we focus on the equatorial Rossby radius of deformation, $\lambda_{R}$, which we express, following Gill (1982), as:

$$
\lambda_{R}=\sqrt{\frac{g H}{2 \beta}},
$$

where $H$ is atmospheric scale height, $\beta=2 \Omega$ represents the Coriolis parameter at the equator, and $g=9.81 \mathrm{~m} \mathrm{~s}^{-2}$. We express scale height as $H=\bar{T}_{s} R / m_{\text {air }} g$, where $\bar{T}_{s}$ is global mean surface temperature and $m_{\text {air }}=0.028 \mathrm{~kg} \mathrm{~mol}^{-1}$ is the molar mass of air (Edson et al. 2011). Letting $a$ be planetary radius, we define slow rotators as planets where $\lambda_{R} / a>1$. Using a present-day Earth GCM, Edson et al. (2011) noted that this definition requires that slow rotators must have a rotational period of $\sim 5$ days or greater, for an Earth-size planet in synchronous orbit around a Gdwarf star. Other studies using more idealized GCMs (Carone et al. 2014, 2015, 2016; Noda et al. 2017) have found comparable values for this limit on slow rotators. All planets in our set of simulations have a rotation period greater than 5 days, except for planets orbiting a $2600 \mathrm{~K}$ host star. This means that, aside from the coolest host star, all these cases should expect to show $\lambda_{R} / a>1$.

The Walker circulation for the set of simulations is shown in Fig. 5, where contours show the strength of the mean zonal circulation, and shading shows rising motion. This particular set of simulations was chosen because they all have an approximately constant value of day-night contrast, where $\left(T_{\text {day }}-T_{\text {night }}\right) / T_{e q} \approx 0.7$. Fig. 5 confirms that $\lambda_{R} / a>1$ for all cases from $4500 \mathrm{~K}$ to 3000 $\mathrm{K}$, where the planet's large scale dynamics are characterized by a thermally direct zonal circulation that spans the day-night hemisphere. All simulations with $\lambda_{R} / a>1$ show the presence of this hemispheric zonal circulation, although only those cases that also show a non-dimensional Rhines 
number (defined in section 4.3) greater than one are classified as slow rotators.

\subsection{Rapid Rotators}

Rapid rotators are characterized by weak convective motion beneath the substellar point, with a Rossby deformation radius less than planetary radius $\left(\lambda_{R} / a<1\right)$ (Fig. 4). Rapid rotators tend to show banded cloud formation beneath the substellar point (Yang et al. 2014; Kopparapu et al. 2016) and a mean zonal circulation that only partially reaches from day to night side (Haqq-Misra \& Kopparapu 2015; Kopparapu et al. 2017) Rapid rotators are comparable to the 'Type-IV' circulation regime described by Noda et al. (2017), with some dynamical features that resemble present-day Earth.

The $2600 \mathrm{~K}$ case appears as an outlier in the bottom right panel of Fig. 2, which can be understood because these planets are in a rapidly rotating regime with a zonal flow that does not span the day-night hemisphere. This is shown in Fig. 5, where the $2600 \mathrm{~K}$ case exhibits its rapid rotation through a less-organized zonal circulation with a larger number of cells that do not span the day-night hemisphere. The $2600 \mathrm{~K}$ case also shows less direct rising motion beneath the substellar point, which leads to a reduction in the amount of convectively-transported water vapor to the upper troposphere.

The set of GCM simulations is plotted in terms of rotation period versus $\lambda_{R} / a$ in Fig. 6 (left panel), which shows that only the $2600 \mathrm{~K}$ case falls within the rapid rotation regime. The rest of the simulations show a thermally-direct circulation that spans day to night hemisphere. Although the $3000 \mathrm{~K}$ case is close to the dashed line in the left panel of Fig. [6] where $\lambda_{R} / a=1$, we can confidently exclude the $3000 \mathrm{~K}$ case from being a rapid rotator, because its Walker circulation spans from day to night side (Fig. [5). Only when the rotation rate is less than $\sim 5$ days (for Earth-mass planets) does the Walker circulation show evidence of the slow rotation dynamical regime.

Some studies have extended their GCM simulations to planets with a smaller rotation period

of 1 day (Merlis \& Scheider 2010; Haqq-Misra \& Kopparapu 2015; Carone et al. 2014, 2015, 2016; Noda et al. 2017). Noda et al. (2017) even discuss a 'Type-III' circulation regime that occurs at a rotation period of a few days, less than the rotational period of our $2600 \mathrm{~K}$ cases. We neglect these cases in our present study because Eq. (1) requires that any planets with a rotation period of 1 day or less that also resides in the habitable zone of its host star must therefore reside around very cool brown dwarf stars. Because our set of calculations extends only to $2600 \mathrm{~K}$ stars, such planets with short 1 day or less orbital periods are beyond the scope of this study.

\subsection{Rhines Rotators}

Rhines rotators are characterized by strong upper-atmosphere superrotation as well as strong upwelling beneath the substellar point, occupying a transition region between slow and rapid ro- 
tators (Fig. 4, lower-right quadrant). Rhines rotators still show a Walker circulation that spans from day to night side (Fig. 5), similar to slow rotators, but their atmospheric dynamics are also characterized by asymmetric zonal jets at midlatitudes. Rhines rotators are analogous to the 'TypeII' circulation regime described by Noda et al. (2017), which show the point of maximum heating beginning to drift off-center from the substellar point. This transitional regime was also noted by Edson et al. (2011) and Carone et al. (2014), which shows an increasing effect of equatorial superrotation dynamics.

Fig. 7 shows surface temperature and wind vectors for the same set of GCM simulations as in Fig. 5. The $4500 \mathrm{~K}, 4000 \mathrm{~K}$, and $3700 \mathrm{~K}$ cases all show characteristic flow inward toward the substellar point, with the surface flow in one hemisphere a mirror image of the other. This mirror symmetry begins to break in the $3300 \mathrm{~K}$ case, with the beginnings of an eastward shift of the point of maximum heating. The $3000 \mathrm{~K}$ and $2600 \mathrm{~K}$ cases both show a notably different flow pattern along the surface and toward the substellar point, with turbulent features contributing to both the midlatitude and equatorial flow. In particular, the vortex-like structures at midlatitude and polar latitudes of the $3000 \mathrm{~K}$ case in Fig. 7 indicate the breaking of symmetry due to turbulent flow.

We further detail the energy distribution of our simulation set by examining the temperature difference between the substellar point at the equator and the pole. Let $T_{\text {equator }}$ be the maximum temperature beneath the substellar region and $T_{\text {pole }}$ be the minimum of the north and south pole temperatures. Fig. 8 shows the equator-to-pole temperature contrast $\left(T_{\text {equator }}-T_{\text {pole }}\right) / T_{\text {eq }}$ versus relative stellar flux, mean surface temperature, the day-night greenhouse effect difference, and the vertically-integrated night side static energy flux convergence. All the slow rotators from $4500 \mathrm{~K}$ through $3300 \mathrm{~K}$ show similar trends as Fig. 2, with the magnitude of equator-to-pole temperature contrast even greater than the magnitude of day-night temperature contrast. By comparison, the $3000 \mathrm{~K}$ and $2600 \mathrm{~K}$ cases appear as outliers from the others in the two panels on the bottom row of Fig. 8, which is due in part to their warmer poles compared to slow rotating cases. This behavior cannot be explained by the Rossby deformation radius alone, since the $3000 \mathrm{~K}$ case shows $\lambda_{R} / a>1$ while the $2600 \mathrm{~K}$ case is a rapid rotator.

Turbulent structures on Earth and other asynchronously rotating planets tend to elongate in the east-west direction compared to north-south, which Rhines (1975) realized is due to the variation in the Coriolis parameter with latitude. The latitudinal scale at which turbulent flow can organize into zonal jets is known as the Rhines length, which is defined as

$$
L_{R}=\pi \sqrt{\frac{U}{\beta}},
$$

where $U$ is a characteristic root mean squared velocity at the relevant energy-containing scale (Showman et al. 2010, 2013; Vallis 2017). In general, the Rhines scale represents the transition scale between turbulent and wave-driven motion. Turbulent structures on Earth can only grow to sizes limited by the Rhines scale, beyond which Rossby waves dynamics become the primary driver. We define a non-dimensional Rhines length, $L_{R} / a$, which indicates the zonal scale to which 
turbulent structures can grow. On Earth and Jupiter today, $L_{R} / a<1$, thereby implying that any zonal jets that emerge from turbulent energy cannot grow to encompass the entire planetary circumference. For synchronously rotating planets, we can use this non-dimensional Rhines length to determine the conditions under which turbulence-driven zonal jets can grow to planetary scales. For $L_{R} / a>1$, the length scale for the turbulent energy cascade is greater than planetary radius, so we expect atmospheric dynamics to be dominated by the thermally-direct circulation from day to night side, with little contribution from turbulence-driven jets. Conversely, for $L_{R} / a<1$, the length scale for the turbulent energy cascade is smaller than planetary radius, so zonal turbulencedriven jets can form at midlatitudes and cause a departure from symmetry in surface flow. We refer to these synchronously rotating planets, defined by $L_{R} / a<1$ and $\lambda_{R} / a>1$, as Rhines rotators.

Fig. 6 (right panel) shows the ratio of the day-night temperature contrast to the equatorto-pole temperature contrast ratio, $\left(T_{\text {day }}-T_{\text {night }}\right) /\left(T_{\text {equator }}-T_{\text {pole }}\right)$, versus the non-dimensional Rhines number. Our calculation of the Rhines length assumes that $U$ is equal to the area-weighted root mean squared surface wind on the day hemisphere, as shown in Fig. 3, For all the slow rotators from $4500 \mathrm{~K}$ to $3700 \mathrm{~K}$, we see that $L_{R} / a>1$, which implies a large-scale dynamical structure characterized by a symmetric thermally-direct circulation from day to night side (Fig. 5). By contrast, all cases for $2600 \mathrm{~K}$ and $3000 \mathrm{~K}$ stars show $L_{R} / a<1$, which implies that the atmospheric dynamics of such planets include contributions at midlatitudes from turbulent-driven zonal jets that break the symmetry of surface flow when compared to slow rotators. Fig. 6] also shows that the $3300 \mathrm{~K}$ simulation resides in a transition zone between Rhines rotation and slow rotation, where $L_{R} / a \approx 1$.

Fig. 4 indicates the cases in our GCM simulations that fall within the Rhines rotation regime, with $L_{R} / a<1$ and $\lambda_{R} / a>1$. These simulations all show very little spread in the value of $L_{R}$, even though stellar flux varies. The primary feature of planets within the Rhines rotation regime is that the atmosphere responds to an increase in stellar energy by decreasing the day-night to equatorpole temperature contrast ratio (Fig. 6, right panel) by breaking zonal symmetry without changing total kinetic energy by much. By comparison, planets in the slow rotation regime respond to an increase in stellar energy by decreasing the day-night temperature contrast as well as the root mean squared wind speed (Fig 3, right panel), which increases total kinetic energy. Rhines rotators are able to develop zonal structures on the scale of the planetary radius, which induces the development of midlatitude and polar vorticies and other transient dynamical features that break the symmetry of the the thermally-directly model for large-scale circulation.

We show zonal wind and the mean meridional (i.e., Hadley) circulation (MMC) in Fig. 9 for the same set of cases as shown in Figs. 5 and 7 . The left row shows the global quantities of zonal wind and MMC, while the middle and right rows show the eastern and western hemisphere, respectively, from the substellar point. Note that the direction of the Hadley circulation changes sign when comparing the eastern and western hemisphere, as discussed by Haqq-Misra \& Kopparapu (2015). This is a prediction that emerges from the simplified shallow water study of Geisler (1981), which found that the MMC changes direction on either side of a fixed heating source. This suggests that 
hemispheric separation of the MMC should always be examined for synchronously rotating planets, as the global mean MMC tends to cancel out branches with an opposite sign of circulation on either side of the substellar point.

The $4500 \mathrm{~K}$ through $3700 \mathrm{~K}$ cases all show surface flow into the substellar point and a stronger easterly jet in the western hemisphere. By contrast the $2600 \mathrm{~K}$ and $3000 \mathrm{~K}$ cases show strong upper-atmospheric superrotation in both hemispheres. The $2600 \mathrm{~K}$ slow rotators show very strong superrotation at all latitudes and in both hemispheres, which owes to these planets having both $L_{R} / a<1$ and $\lambda_{R} / a<1$. The $3000 \mathrm{~K}$ case is a Rhines rotator with $L_{R} / a<1$ and $\lambda_{R} / a>1$, which manifests as zonal jets at the surface and middle of the atmosphere. We see zonal propagation of Rossby waves dominating the upper-atmosphere of the $3000 \mathrm{~K}$ cases, although an upper-level westerly jet also persists in these simulations. The $3300 \mathrm{~K}$ case remains at the threshold of the Rhines rotator transition, with a strong easterly jet persisting in both hemispheres and a strong westerly jet at the top of the model atmosphere.

The Rhines rotator regime represents a transitional, but distinct, dynamical state for terrestrial planets in synchronous rotation. Rhines rotators respond to an increase in stellar forcing by decreasing the equator-pole temperature contrast and are characterized by the presence of planetary-scale turbulent structures. Rhines rotators should be expected for terrestrial planets in the habitable zone of M-dwarf stars with an effective stellar temperature of $3000 \mathrm{~K}$ to $3300 \mathrm{~K}$.

We also note that the upper-left quadrant of Fig. 4 represents a fourth regime where the Rhines length is larger than the Rossby deformation radius, although this situation did not arise in our simulations. This regime could exist in principle, but most cold atmospheres show a root mean squared velocity of the zonal wind less than the gravity wave speed (implying that the Rhines length is smaller than the Rossby deformation radius). Hot atmospheres, such as closely-orbiting hot Jupiters, could conceivably host supersonic winds that may fall within this regime. However, this fourth regime is probably not feasible within the terrestrial planet habitable zone.

\section{Implications for Observations}

All of our simulations show a strong correlation between the day-night surface temperature contrast and global mean surface temperature, which is driven by the increase in dry static energy flux convergence on the night side (Fig. 2). This decrease in day-night contrast also can be interpreted as an increase in the atmospheric optical thickness, (Eqs. (3) and (4)), which suggests that synchronously rotating planets observed near the inner edge of the habitable zone should show a smaller day-night temperature contrast than planets orbiting farther outward. Planets near the outer edge of the habitable zone should build up a dense carbon dioxide atmosphere (Kasting et al. 1993), which also increases longwave optical depth and thus should decrease the day-night temperature contrast. Observations of the day-night temperature contrast on terrestrial synchronous rotators will provide important constraints on modeling the atmospheres of these 
planets.

The dynamical regime depends upon the Rossby deformation radius and Rhines length. Planetary rotation period can be inferred from observations of orbital period for planets around low mass stars where tidal locking is expected, but the combinations of models and observations will be needed to estimate values of mean wind speed, buoyancy, or other indicators of the Rossby deformation radius and Rhines length. If we assume a fixed Earth-like radius, as with our set of simulations, then we can use the fact that rotation period is also a function of spectral stellar type (Eq. (1)), which allows us to predict the dynamical state of planets in the habitable zone of a given low-mass star. Planets around M-dwarf stars with effective temperatures of $3700 \mathrm{~K}$ to 4500 $\mathrm{K}$ (rotation period $>20$ days) should be slow rotators, with thermally-direct large-scale circulation from the day to night side. Other planets around the lowest mass stars we consider, with effective temperature of less than $3000 \mathrm{~K}$ (rotation period $<5$ ), should be rapid rotators that exhibit strong upper-level jets with asymmetric flow in the lower troposphere. Stars with effective temperatures in the range of $3000 \mathrm{~K}$ to $3300 \mathrm{~K}$ (rotation period $\sim 5$ to 20 ) represent the intermediate state of Rhines rotators, which exhibit planetary-scale turbulent flow while still retaining a thermally-direct circulation from the day to night side.

We can apply our model results to think about the expected dynamical regime for recently discovered terrestrial-sized exoplanets, assuming synchronous rotation. The TRAPPIST-1 systems may contain several planets within the traditional liquid water habitable zone, with TRAPPIST-1e being the most promising candidate (Wolf 2017; Turbet et al. 2017). With an orbital period of $\sim 6$ days, TRAPPIST-1e would be close to the rapid rotator regime - especially if its lower planetary mass implies a lower atmospheric scale height (and thus a smaller value of $\lambda_{R}$ ). Proxima Centauri $\mathrm{b}$ has an orbital period of $\sim 11$ days, which places it within the Rhines rotation regime if it is able to sustain an atmosphere. The planet LHS 1140b has a slower rotation period of $\sim 24$ days, which implies that its surface flow and heating should exhibit the near-symmetry of the slow rotation regime. These conclusions not only assume that these planets are in synchronous rotation but also that the atmospheric dynamics can be approximated by our 1-bar atmosphere simulations with an Earth-sized planet.

These dynamical regimes can also be extended to planets with a larger radius than Earth. A larger planet has a faster rotation about its axis compared to a smaller planet with equal angular velocity. For example, a planet with Earth-like terrestrial features but a radius twice as large as Earth would be a slow rotator with rotation period less than 10 days and a Rhines rotator with rotation period from $\sim 10$ to 40 days. We also note that changes in atmospheric thickness and composition could also affect the Rossby deformation radius and Rhines number, and therefore the characterization of the atmosphere's dynamical state. Further GCM studies that explore the mass and radius dependence of these dynamical regimes will place better constraints on known and future synchronously rotating exoplanets.

Thermal phase curves have been proposed as a relatively simple method for observing and 
characterizing terrestrial extrasolar planets (e.g., Cowan et al. (2012)). Thermal phase curves show the disk-integrated thermal flux emitted by a planet as seen by the observer as a function of the planet's position in its orbit around the host star. Assuming an (approximately) edge on orientation, a phase angle of $\pm 180^{\circ}$ corresponds with a transit event, when only the night side of the planet is in the field of view of the observer. A phase of angle of $0^{\circ}$ corresponds with the secondary eclipse, where only the day side of the planet is visible to the observer (excluding the actual secondary eclipse event of course). JWST will observe across most infrared wavelengths, and may be able to resolve the shape of thermal phase curves for terrestrial planets in nearby M-dwarf systems (Meadows et al. 2016).

We calculate broadband thermal emission phase curves following the method of Koll \& Abbot (2015), using outgoing thermal flux maps produced by our GCM simulations, time averaged over many orbits. In Fig. 10, we show thermal phase curves for 6 different simulations. Note that the simulations shown in Fig. 10 are the same set as shown in Figs. 5, 7, and 9, Each of the three dynamical regimes are clearly evident and differentiable within broadband thermal emission phase curves. The differences between thermal phase curves for rapid, slow, and Rhines rotators are a result of the interaction of atmospheric dynamics with clouds and water vapor, which in turn feedback on both the planetary surface temperature and the allowed outgoing thermal radiation at the top of the atmosphere.

For each case, the modulation of the upwelling longwave flux (and thus the thermal emission phase curve) is tied closely to the location of high-altitude water ice (i.e., cirrus) clouds (Fig. 11). Cirrus clouds are efficient absorbers of longwave radiation emitted by the planet surface (Ramanathan et al. 1989). Fig. 11 shows the water ice cloud condensate mass mixing ratio, specific humidity, upwelling longwave flux, and upwelling longwave clearsky flux for each dynamical regime, with all atmospheric columns located along the equator. Note that the substellar point is at a longitude of $0^{\circ}$ and the antistellar point is a longitude of $\pm 180^{\circ}$. The bottom panel of Fig. 11 shows that if we omit clouds from the radiative transfer calculation, then the upwelling longwave clearsky flux is generally uniform. This indicates that cirrus clouds, rather than the advection of water vapor and its associated greenhouse effect, are responsible for the morphology of the thermal phase curves.

Unsurprisingly, for all cases studied the hottest planetary surface temperatures are found on the day side (Fig. 7). In the absence of an atmosphere, or when the greenhouse effect is perfectly uniform everywhere, the thermal emission phase curve should mirror the disk integrated surface temperature distribution as a function of phase angle. However, cirrus clouds ultimately control where thermal energy can be effectively emitted to space. Rapid rotators exhibit a maximum in thermal emission when viewing the day side of the planet. Clouds on rapid rotating worlds are advected eastward off of the substellar point by the strongly super-rotating atmosphere. A minimum in the thermal emission is coincident with the primary cirrus cloud deck that is located near the eastern terminator of the planet (Fig. 11). Locations immediately west of the substellar point have relatively few clouds (e.g., Kopparapu et al. (2017) Fig. 8), resulting in both warmer 
surface temperatures and efficient emission of thermal energy to space. Thus, the maximum in the thermal phase curve for rapid rotators is shifted $\sim 45^{\circ}$ west of the substellar point (Fig. 10), where surface temperatures are warm and clouds are absent.

For slow rotators again the surface temperature maximum is found at the substellar point; however thick and symmetric clouds completely enshroud the day side hemisphere. A thick cap of high altitude cirrus clouds significantly reduces the outgoing thermal flux from the day side by lowering the emitting temperature of the atmosphere (Fig. 11). The night side remains cloud free and can efficiently radiative energy to space. The night side thermal emission remains large despite cold surface temperatures, due to the near-surface inversion layer described in section 3 and the low water vapor concentration, which allows the night side to act as a 'radiator fin' that emits excess thermal energy to space similar to the dry subtropics of Earth (Yang \& Abbot 2014). This results in a minimum in the thermal phase curve when viewing the day side of the planet and maximum when viewing the night side for slow rotators, as first noted by Yang et al. (2013). Note that the thermal phase curve for slow rotators exhibits remarkable symmetry with minima at $0^{\circ}$ and maxima at $\pm 180^{\circ}$, due to the symmetric day-night general circulation that occurs on these worlds. We also note that ocean heat transport will affect these results, as surface fluxes induces by the ocean can be significant and can also depend upon planetary rotation period (Cullum et al. 2014; Hu \& Yang 2014; Way et al. 2016), although the presence of continents could limit the magnitude of ocean heat transport (Yang et al. 2013).

While differences between fast and slow rotators are evidently clear, the differentiation of Rhines rotators is more subtle. The thermal phase curve for Rhines rotators is qualitatively similar to that of slow rotators, however the minimum is shifted $\sim 45^{\circ}$ westward of the substellar point. Tropospheric water clouds still generally permeate the substellar hemisphere of Rhines rotators, keeping albedos large and suppressing the surface temperature. However, the primary ice cloud layer is shifted westward of the substellar point, shifting the associated minimum in the thermal phase curve accordingly. This corresponds to the strong westerly jet present in the uppermost model layers of this Rhines rotator (Fig. 9, middle row), which is nonexistent in the slow rotators and extremely weak in the rapid rotators. Rhines rotators may be differentiated from slow rotators by the offset of the minimum in the thermal phase curve. Our results imply that understanding stratospheric process and ice cloud formation on terrestrial extrasolar planets may be critical for interpreting observed thermal phase curves, although we caution that further studies with other GCMs, using different ice cloud parameterizations, will be needed to demonstrate robustness of these phase curve features.

\section{Conclusion}

Our examination of the atmospheres of planets in synchronous rotation around low mass stars reveals three distinct dynamical regimes (Fig. 4). Rapid rotation occurs when the Rossby deformation radius is less than planetary radius (rotation period $>5$ days). Slow rotation occurs 
when both the Rossby deformation radius and the Rhines length are greater than planetary radius (rotation period $>20$ days). Rhines rotation occurs when the Rhines length is less than planetary radius but the Rossby deformation radius is greater than planetary radius, which allows turbulent structures to reach planetary scales (rotation period $\sim 5$ to 20 days).

These three dynamical states can be distinguished from one another through observations of the thermal phase curve of the planet. Differences in the amplitude of the maxima and minima of the thermal phase curves can be used to identify the slow rotation regime, while the transition between the rapid rotation and Rhines regimes can be identified by comparing the morphology of the thermal phase curves. Corroborating these phase curves with observations of the planet's orbital period and the host star's spectral type will provide a basis for further characterization of such atmospheres with computational models.

We also show that the day-night surface temperature contrast for terrestrial planets decreases as incident stellar flux increases. The combined effects of moisture accumulation and the increase in static energy flux divergence on the night side leads to an increase in greenhouse effect that diminishes the temperature contrast between the day and night sides. We therefore expect that synchronously rotating planets near the inner edge of the habitable zone should have diminished day-night surface temperature contrasts compared with other synchronous rotators at farther orbital distances.

We can apply these results to the response of a synchronously rotating atmosphere under the steady main sequence brightening of its host star. Planets in the slow rotation regime respond to an increase in stellar forcing with a decrease in both the day-night temperature contrast and the root mean squared surface wind, whereas planets in the Rhines and slow rotation regimes respond to a similar increase by decreasing the day-night to equator-pole temperature contrast ratio. This suggests that the atmospheres of slow rotators will adapt through reducing the equatorial daynight temperature contrast with an increase in static energy flux convergence on the night side. Rhines and slow rotators will react by increasing the equator-pole contrast more than the day-night contrast, tending toward a surface temperature distribution with even heating at all latitudinal bands. It is beyond the scope of this paper to speculate as to which of these three regimes, if any, would be conducive or adverse to the presence of life. But we can at least begin to think about how the evolution of a low mass star can affect the dynamical state of a planet within its habitable zone.

The authors thank Jun Yang for constructive comments that greatly improved the manuscript. J.H, E.T.W., and R.K.K. acknowledge funding from the NASA Habitable Worlds program under award NNX16AB61G. R.K.K. also acknowledges funding from NASA Astrobiology Institute's Virtual Planetary Laboratory lead team, supported by NASA under cooperative agreement NNH05ZDA001C. X.Z. acknowledges support from NSF grant AST1740921. This work was facilitated though the use of advanced computational, storage, and networking infrastructure provided by the Hyak supercomputer system at the University of Washington. This work benefited from the Exoplanet 
Summer Program in the Other Worlds Laboratory (OWL) at the University of California, Santa Cruz, a program funded by the Heising-Simons Foundation. Any opinions, findings, and conclusions or recommendations expressed in this material are those of the authors and do not necessarily reflect the views of NASA or NSF.

\section{A. Static energy flux convergence by isallobaric wind}

As stellar flux increases, slow rotators and Rhines rotators show a decrease in day-night temperature contrast that corresponds with an increase in the night (day) side static energy flux convergence (divergence). We demonstrate here that this relationship is ultimately driven by increases in the night (day) side convergence (divergence) of the component of wind driven by pressure contrasts known as the isallobaric wind.

Let $\mathbf{v}$ be the horizontal wind vector and $s=c_{p} T+\Phi+L_{v} q$ be the moist static energy per unit mass. The static energy flux divergence can be written as

$$
\nabla \cdot(\mathbf{v} s)=s \nabla \cdot \mathbf{v}+\mathbf{v} \cdot \nabla s
$$

where the first term on the right is horizontal wind divergence, and the second term on the right is advection of static energy. Static energy divergence refers specifically to the situation when $\nabla \cdot(\mathbf{v} s)>0$, while static energy convergence implies that $\nabla \cdot(\mathbf{v} s)<0$. We are primarily concerned with comparing $\nabla \cdot(\mathbf{v} s)$ between day and night sides, where the day side is characterized by heating

across much of the hemisphere and the night side is characterized by near-uniform cold temperatures across most of the hemisphere. This suggests that we can simplify Eq. (A1) by assuming $\nabla s \approx 0$ for these atmospheres.

The geostrophic wind describes the balance between the pressure gradient force and Coriolis force, which we can write as

$$
\mathbf{v}_{g}=\mathbf{k} \times \frac{1}{\rho f} \nabla p,
$$

where $\mathbf{k}$ is the upward unit vector, $f$ is the Coriolis parameter, $\rho$ is air density, and $p$ is pressure. However, we cannot substitute $\mathbf{v}_{g}$ for $\mathbf{v}$ in Eq. (A1) because the geostrophic wind is non-divergent $\left(\nabla \cdot \mathbf{v}_{g}=0\right)$. We instead consider the quasigeostrophic approximation, where the wind vector is the sum of geostrophic and ageostrophic components, $\mathbf{v}=\mathbf{v}_{g}+\mathbf{v}_{a}$. The quasigeostrophic approximation allows us to express the ageostrophic wind as

$$
\begin{array}{rlc}
\mathbf{v}_{a} & = & \frac{1}{f} \mathbf{k} \times \frac{D \mathbf{v}_{g}}{D t} \\
& =\frac{1}{f} \mathbf{k} \times \frac{\partial \mathbf{v}_{g}}{\partial t}+\frac{1}{f} \mathbf{k} \times\left(\mathbf{v}_{g} \cdot \nabla \mathbf{v}_{g}\right)
\end{array},
$$

following Holton (2004). The two terms on the bottom line of Eq. (A3) respectively represent the isallobaric and advective components of the ageostrophic wind. The advective term can contribute to ageostrophic wind divergence through positive vorticity advection; however, vorticity advection 
between day and night hemispheres is small. We therefore focus our analysis on the contribution of the isallobaric wind to the static energy flux.

The isallobaric wind is perpendicular to lines of constant geopotential tendency (known as isallobars) and describes flow toward regions of falling pressure. Substituting from Eq. (A2), we write the isallobaric wind, $\mathbf{v}_{i s}$, as

$$
\mathbf{v}_{i s}=\frac{-1}{f^{2} \rho} \nabla\left(\frac{\partial p}{\partial t}\right)
$$

We now replace the total wind vector in Eq. (A1) with the isallobaric wind in Eq. (A4) to obtain

$$
\nabla \cdot(\mathbf{v} s) \approx s \nabla \cdot \mathbf{v}_{i s}=\frac{-s}{f^{2} \rho} \nabla^{2}\left(\frac{\partial p}{\partial t}\right)
$$

Eq. (A5) shows that an increase (decrease) in pressure corresponds to divergence (convergence) of the isallobaric wind. For synchronous rotators, the increase in pressure due to fixed stellar heating on a single hemisphere causes the isallobaric wind, as well as the static energy flux, to diverge on the day side and converge on the night side, which decreases the day-night temperature contrast.

\section{REFERENCES}

Allard, F., Allard, N. F., Homeier, D., et al., 2007, A\&A, 474, L21-L24

Anglada-Escudé, G., Amado, P. J., Barnes, J., et al., 2017, Nature, 536, 437

Bintanja, R., Graversen, R. G., \& Hazeleger, W., 2011, Nature Geosci., 4, 758

Carone, L., Keppens, R., \& Decin, L., 2014, MNRAS, 445, 930

Carone, L., Keppens, R., \& Decin, L., 2015, MNRAS, 452, 2413

Carone, L., Keppens, R., \& Decin, L., 2016, MNRAS, 461, 1981

Cowan, N. B., Voigt, A., \& Abbot, S., 2012, ApJ, 757, 80

Cullum, J., Stevens, D., \& Joshi, M., 2014, Astrobiol., 14, 645

Curry, J. A., Schramm, J. L., Rossow, W. B., \& Randall, D., 1996, J. Clim., 9, 1731.

Dole S. H., 1964, Habitable Planets for Man, (Blaisdell: New York)

Edson, A., Lee, S., Bannon, P., Kasting, J. F., \& Pollard, D., 2011, Icarus, 212, 1

Fujii, Y., Del Genio, A. D. \& Amundsen, D. S., 2017. arXiv preprint, arXiv:1704.05878

Geisler, J. E., 1981, J. Atmos. Sci., 38, 1390 
Gillon, M., Triaud, A. H., Demory, B. O., et al., 2017, Nature, 542, 456

Gill, A., 1982, Atmospheric-Ocean Dynamics. Int. Geophys. Ser., 30.

Haberle, R. M., McKay, C. P., Tyler, D. \& Reynolds, R. T., 1996, in Circumstellar Habitable Zones, ed. L. R. Doyle, (Travis House: Menlo Park, CA)

Haqq-Misra, J. \& Kopparapu, R. K., 2015, MNRAS, 446, 428

Hu, Y. \& Yang, J., 2014, PNAS, 111, 629

Joshi, M. M., Haberle, R. M., Reynolds, R. T., 1997, Icarus, 129, 2

Joshi, M. M. 2003, Astrobiol., 3, 415

Kasting, J. F., Whitmire, D. P., \& Reynolds, R. T. 1993, Icarus, 101, 108

Koll, D. D. B. \& Abbot, D. S., 2015, ApJ, 802, 21

Koll, D. D. B. \& Abbot, D. S., 2016, ApJ, 825, 99

Komacek, T. D. \& Showman, A. P., 2016, ApJ, 82, 16

Komacek, T. D., Showman, A. P., \& Tan, X., 2017, ApJ, 835, 198

Kopparapu, R. K., Ramirez, R., Kasting, J. F., et al., 2013, ApJ, 765, 131

Kopparapu, R. K., Ramirez, R., SchottelKotte, J., et al., 2014, ApJ, 787, L29

Kopparapu, R. K., Wolf, E.T., Haqq-Misra, J., et al., 2016, ApJ, 819, 1

Kopparapu, R. K., Wolf, E.T., Arney, G., et al., 2017, ApJ, 845, 5

Leconte, J., Forget, F., Charnay, B., et al., 2013, A\&A, 554, A69

Liu, Y., Key, J. R., Schweiger, A., \& Francis, J., 2006, J. Clim., 19, 4902.

Meadows, V. S., Arney, G. N., Schwieterman, E., et al., 2016, Astrobiol., in review

Merlis, T. \& Schneider, T., 2010, J. Adv. Model. Earth Sys. 2, 13

Noda, S., Ishiwatari, M., Nakajima, K., et al., 2017, Icarus, 282, 1

Perez-Becker, D. \& Showman, A. P., 2013, ApJ, 776, 134

Ramanathan, V., Cess, R. D., Harrison, E. F., et al., 1989, Science, 243, 57

Rhines, P. B., 1975, J. Fluid Mech., 69, 417

Showman, A. P. \& Guillot, T., 2002, A\&Aj, 385, 166 
Showman, A. P., Cho, J. Y.-K., \& Menou, K., 2010, in Exoplanets, ed. S. Seager (University of Arizona Press), 471

Showman, A. P., Wordsworth, R. D., Merlis, T. M., \& Kaspi, Y., 2013, in Comparative Climatology of Terrestrial Planets, ed. S. J. Mackwell et al. (University of Arizona Press), 277

Selsis, F., Kasting, J. F., Levrard, B., et al., 2007, A\&A, 476, 1373

Selsis, F., Wordsworth, R. D., \& Forget, F., 2011, A\&A, 532, A1

Turbet, M, Bolmont, E., Leconte, J, et al., 2017, A\&A, in review.

Vallis, G. K., 2017, Atmospheric and Oceanic Fluid Dynamics: Fundamentals and Large-Scale Circulation (2nd ed; Cambridge University Press)

Wordsworth, R., 2015, ApJ, 806, 180

Way, M. J., Del Genio, A. D., Kelley, M., et al., 2015, Comparative Climatology of Terrestrial Planets II, NASA Conference Proceeding technical No. TBD

Wolf, E. T., 2017, ApJ, 839, L1

Yang, J., Cowan, N. B., \& Abbot, D. S., 2013, ApJ, 771, L45

Yang, J., Boué, G., Fabrycky, D. C., \& Abbot, D. S., 2014, ApJ, 787, L2

Yang, J., \& Abbot, D. S., 2014, ApJ, 784, 155.

Zhang, X. \& Showman, A. P., 2017, ApJ, 836, 73 

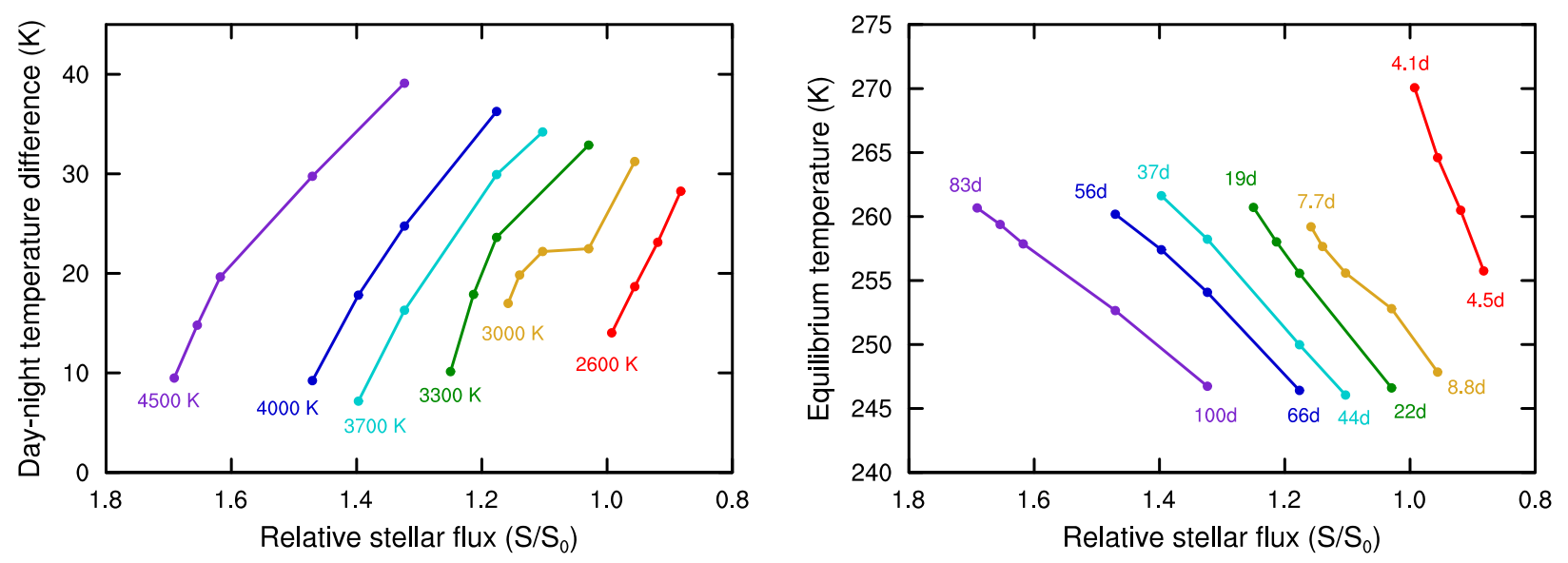

Fig. 1. - The full set of simulations is shown as a function of relative stellar flux, $S / S_{0}$, with labels indicating the stellar effective temperature of the host star (left) and the rotation period of the planet (right), and warmer stars at the left side of the panel. As the model atmosphere warms due to an increase in $S / S_{0}$, the day-night temperature difference, $T_{d a y}-T_{n i g h t}$, decreases (left), while equilibrium temperature, $T_{e q}$, increases (right). 

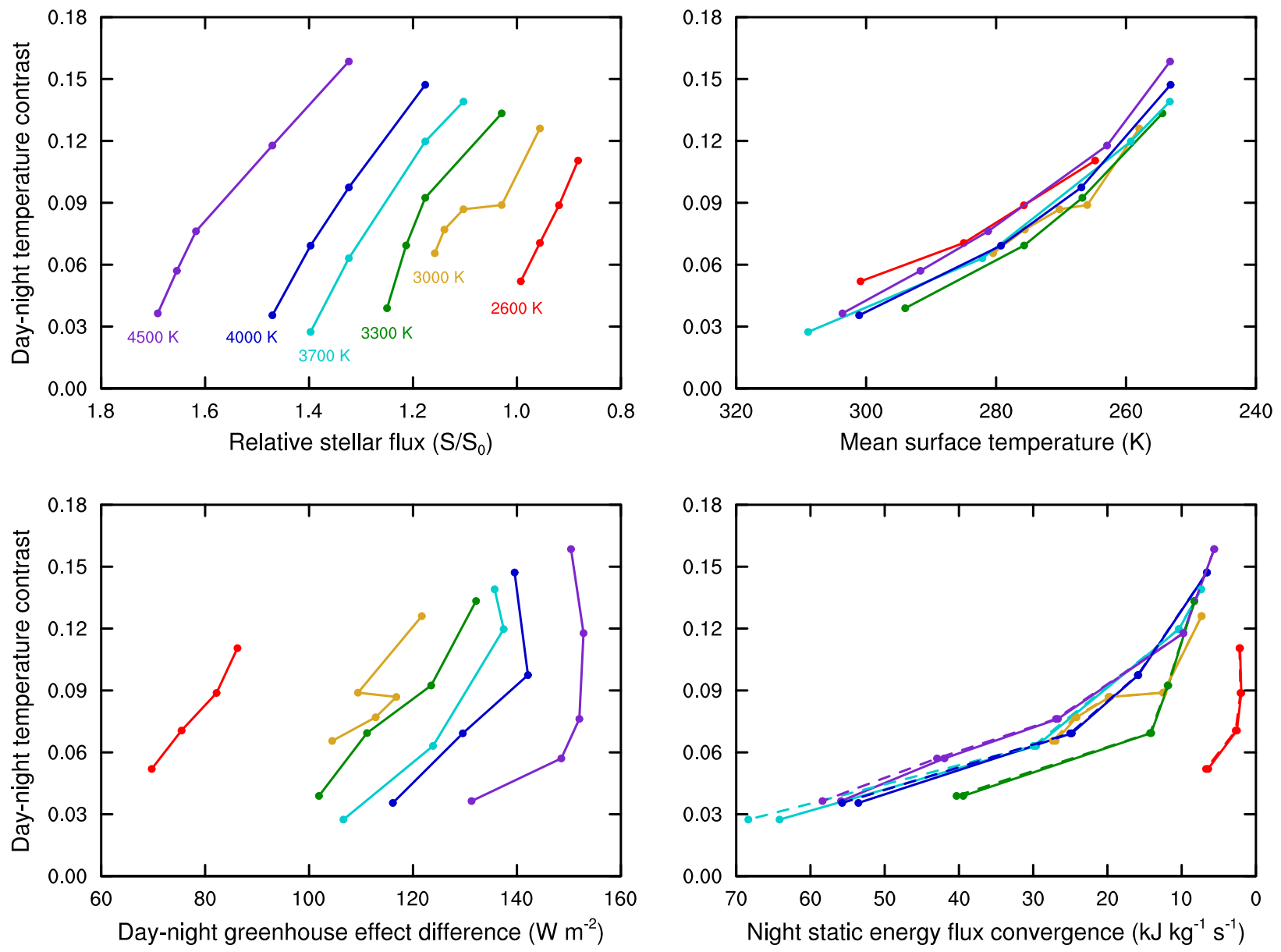

Fig. 2.- The full set of simulations is shown as scaled day-night temperature contrast, $\left(T_{d a y}-\right.$ $\left.T_{\text {night }}\right) / T_{\text {eq }}$, versus relative stellar flux, $S / S_{0}$, with warmer stars at the left side of the panel (top left). These simulations all show a correlation between scaled day-night temperature contrast and mean surface temperature (top right). This decrease in day-night temperature contrast as the planet warms corresponds to a decrease in the day-night greenhouse effect difference (bottom left), which occurs as a result of an increase in the the dry static energy flux convergence on the night side (bottom right, solid lines). The total static energy, with the moist latent energy component included, is also shown (bottom right, dashed lines), but the contribution of this latent energy is very small. 

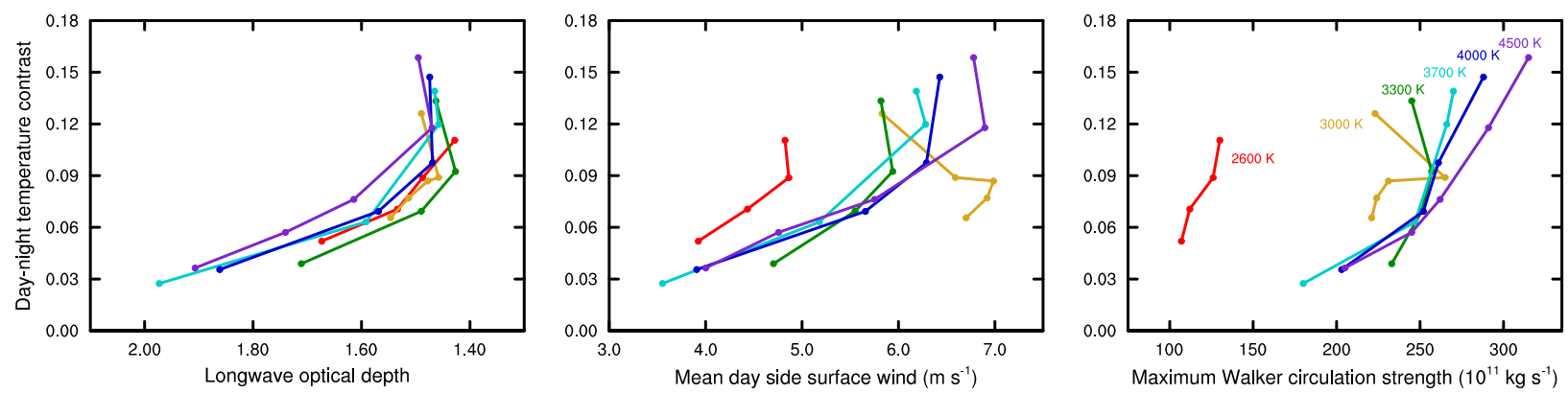

Fig. 3. - The longwave equivalent gray optical depth calculated from Eqs. (3) and (44) shows that these model atmospheres become increasingly optically thick as the planet warms and the daynight temperature contrast shrinks (left). The mean day side surface wind (middle) and maximum strength of the Walker circulation (right) both tend to decrease as the planet warms. Note that the $2600 \mathrm{~K}$ and $3000 \mathrm{~K}$ cases show significant differences from the other simulations.

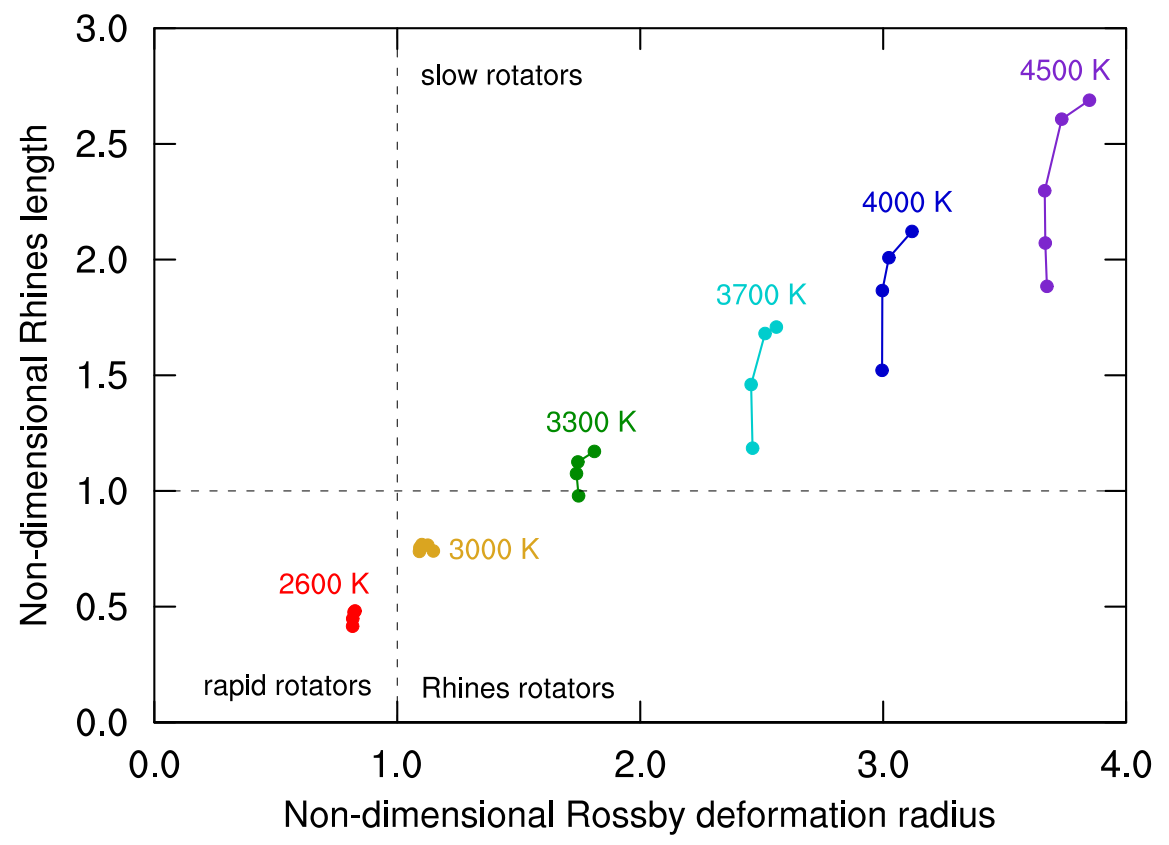

Fig. 4.- Synchronously rotating planets are in the rapid rotation regime when the non-dimensional Rossby deformation radius is less than one, $\lambda_{R} / a<1$, which includes all $2600 \mathrm{~K}$ simulations. The Rhines rotation regime occurs when the non-dimensional Rhines length is less than one but the non-dimensional Rossby deformation radius is greater than one, $L_{R} / a<1$ and $\lambda_{R} / a>1$, which includes all $3000 \mathrm{~K}$ simulations and one $3300 \mathrm{~K}$ case. Planets in the slow rotation regime have both $\lambda_{R} / a>1$ and $L_{R} / a>1$, which describes the remaining simulations for stars $3300 \mathrm{~K}$ to $4500 \mathrm{~K}$. 

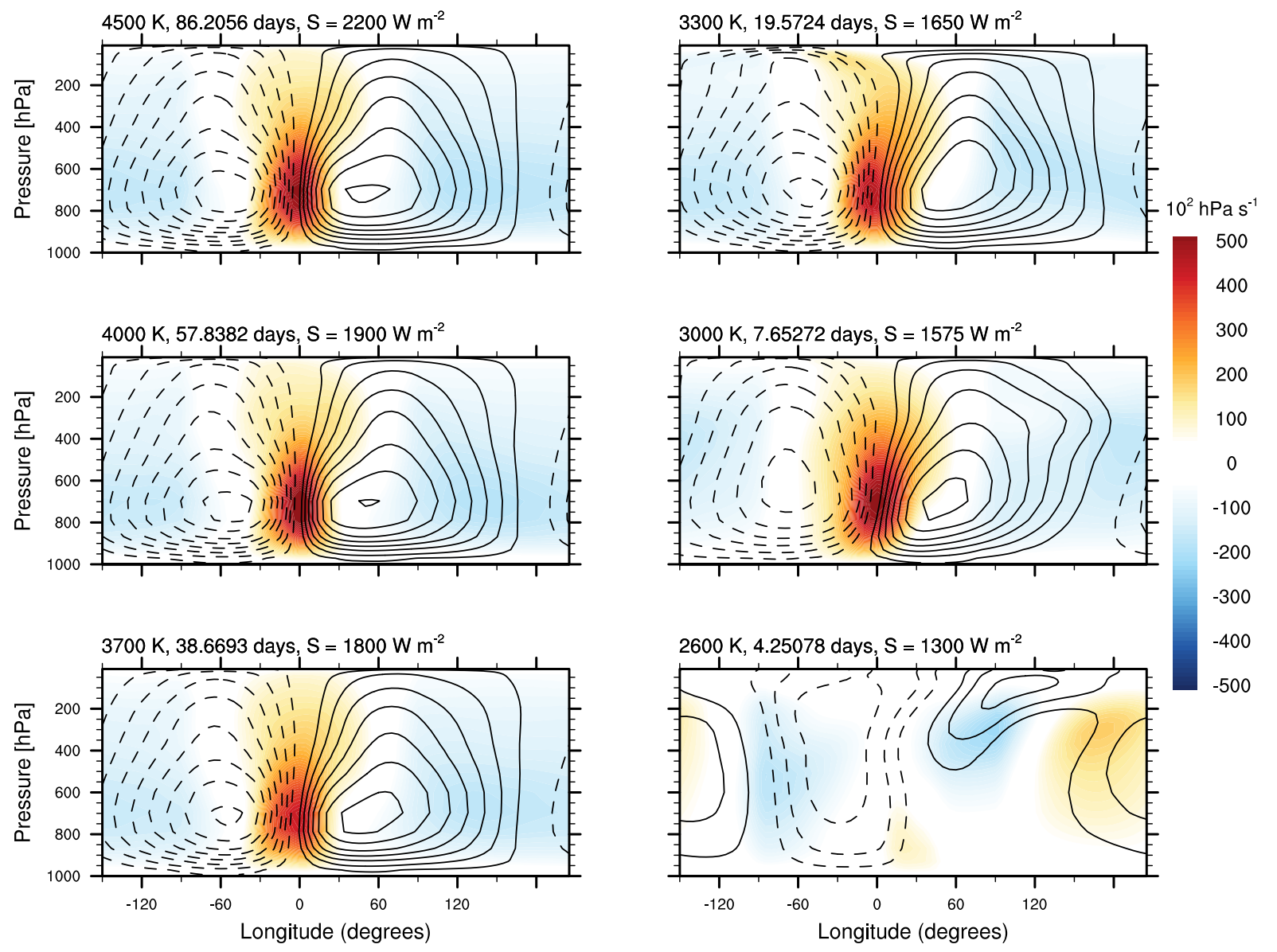

Fig. 5.- The Walker circulation and meridionally-averaged vertical wind for synchronously rotating planets around $4500 \mathrm{~K}, 4000 \mathrm{~K}, 3700 \mathrm{~K}, 3300 \mathrm{~K}$, and $3000 \mathrm{~K}$ stars all show a thermally direct zonal circulation that spans day to night side. The $2600 \mathrm{~K}$ case shows rapid rotation with a Walker circulation that spans less than a full hemisphere. The contour interval for the Walker circulation is $30 \times 10^{11} \mathrm{~kg} \mathrm{~s}^{-1}$, with solid contours indicating clockwise circulation and dashed contours indicating counterclockwise circulation. Shading indicates pressure tendency, which corresponds to rising (warm colors) or sinking (cool colors) motion. 

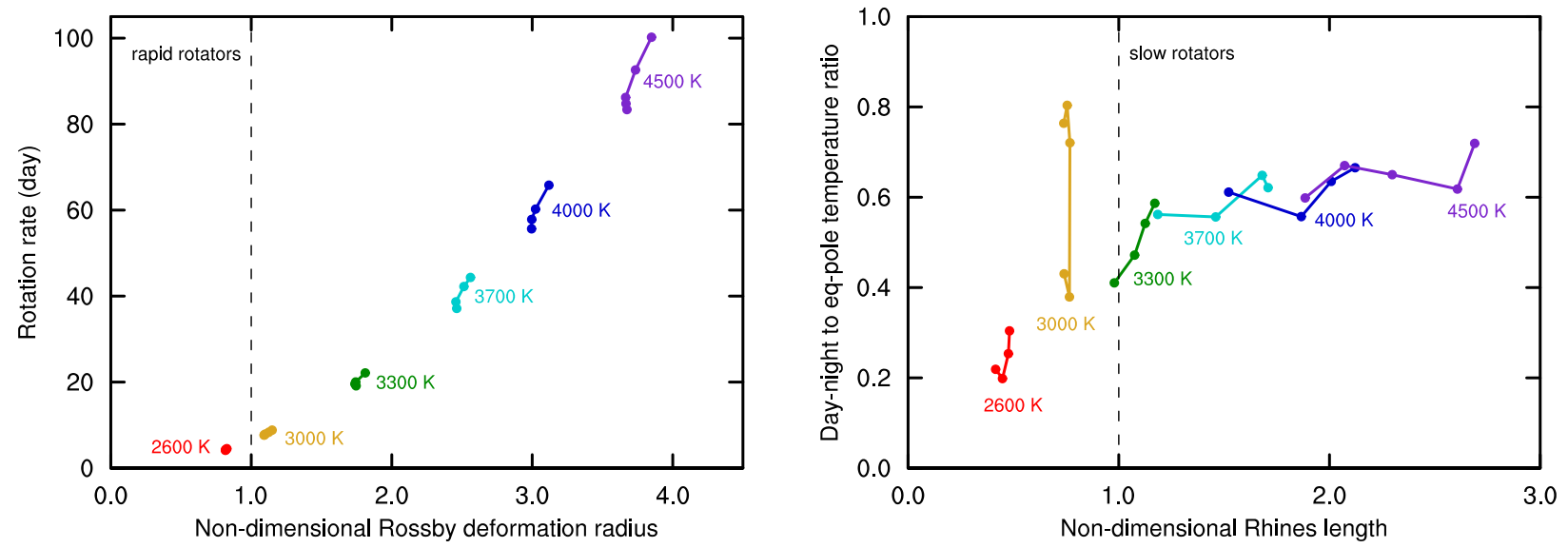

Fig. 6.- Synchronously rotating planets are in the rapid rotation regime when the non-dimensional Rossby deformation radius is less than one, $\lambda_{R} / a<1$, which occurs at a rotation period of $\sim 5$ days (left). The Rhines rotation regime occurs when the non-dimensional Rhines length is less than one, $L_{R} / a<1$ (right). Such planets respond to an increase in stellar flux by reducing the day-night temperature to equator-to-pole temperature ratio, $\left(T_{\text {day }}-T_{\text {night }}\right) /\left(T_{\text {equator }}-T_{\text {pole }}\right)$. Planets in the slow rotation regime have both $\lambda_{R} / a>1$ and $L_{R} / a>1$. 

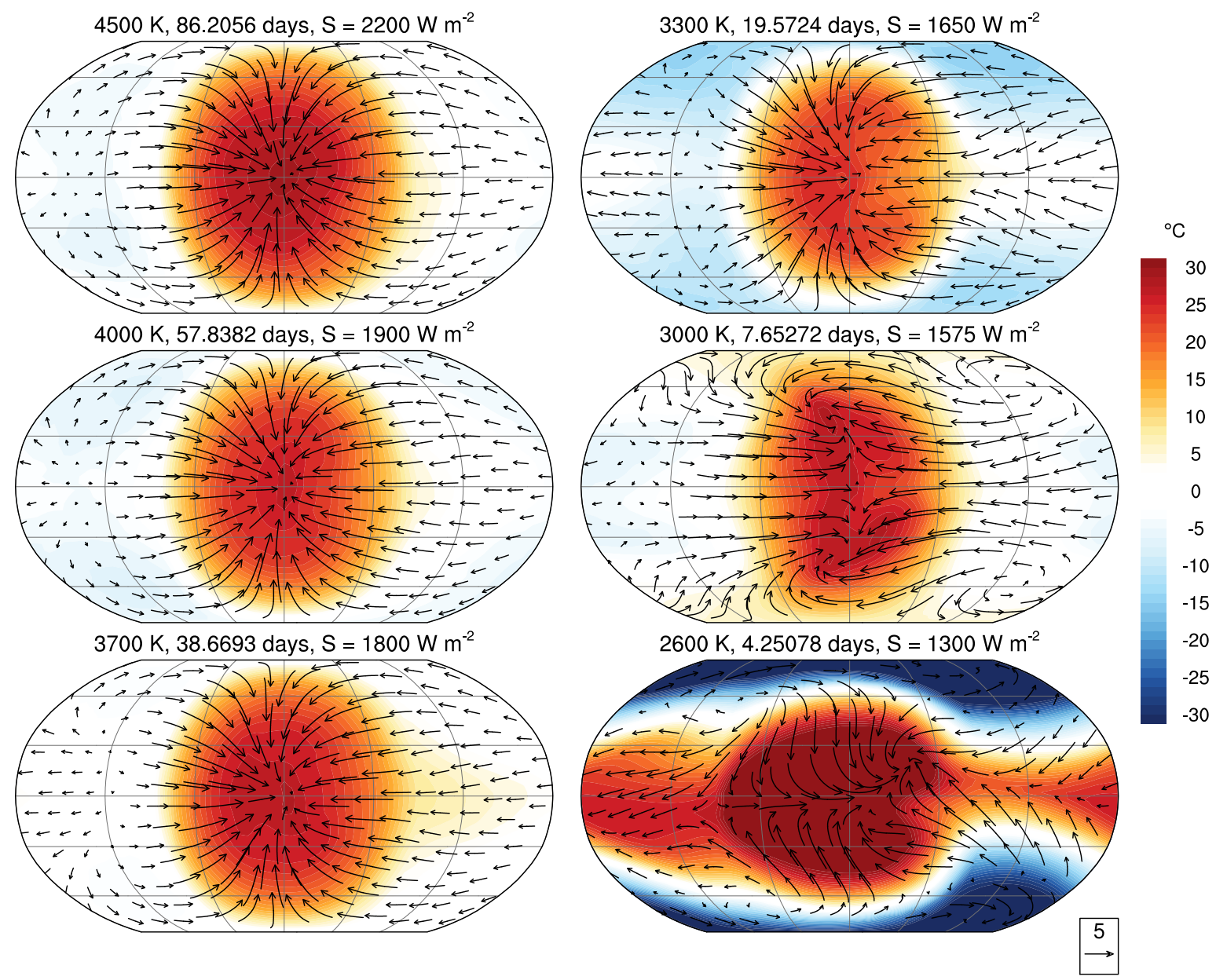

Fig. 7.- Surface temperature and surface wind vectors show a nearly symmetric pattern of substellar heating and flow toward the substellar point for planets in the slow rotation regime around $4500 \mathrm{~K}, 4000 \mathrm{~K}, 3700 \mathrm{~K}$, and $3300 \mathrm{~K}$ stars. The $2600 \mathrm{~K}$ case in the rapid rotation regime shows asymmetric warming flow patterns that extend in an equatorial band from day to night side. In between is the Rhines regime, which shows departure from symmetry particularly at midlatitudes for the $3000 \mathrm{~K}$ cases. 

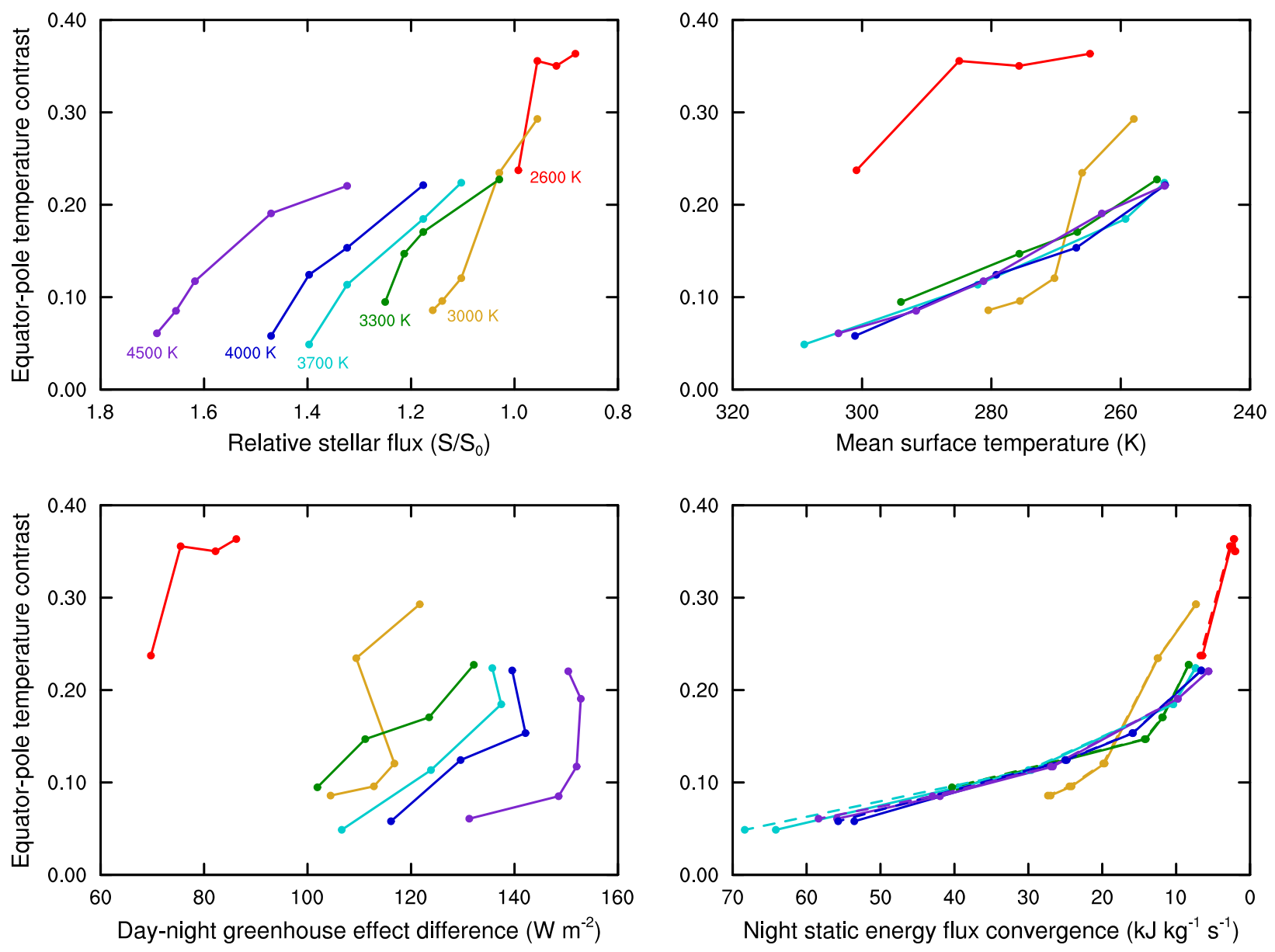

Fig. 8.- The full set of simulations is shown as scaled equator-to-pole temperature contrast, $\left(T_{\text {equator }}-T_{\text {pole }}\right) / T_{\text {eq }}$, versus relative stellar flux, $S / S_{0}$, with warmer stars at the left side of the panel (top left). Simulations in the slow rotation regime show a correlation between scaled equatorto-pole temperature contrast and mean surface temperature (top right), which corresponds with the decrease in day-night greenhouse effect difference (bottom left) that occurs in response to the increase in night side dry (solid) and moist (dashed) static energy flux convergence (bottom right). The $2600 \mathrm{~K}$ rapid rotation and $3000 \mathrm{~K}$ Rhines rotation cases show notably different behavior due in part to their warmer poles compared to slow rotators. 

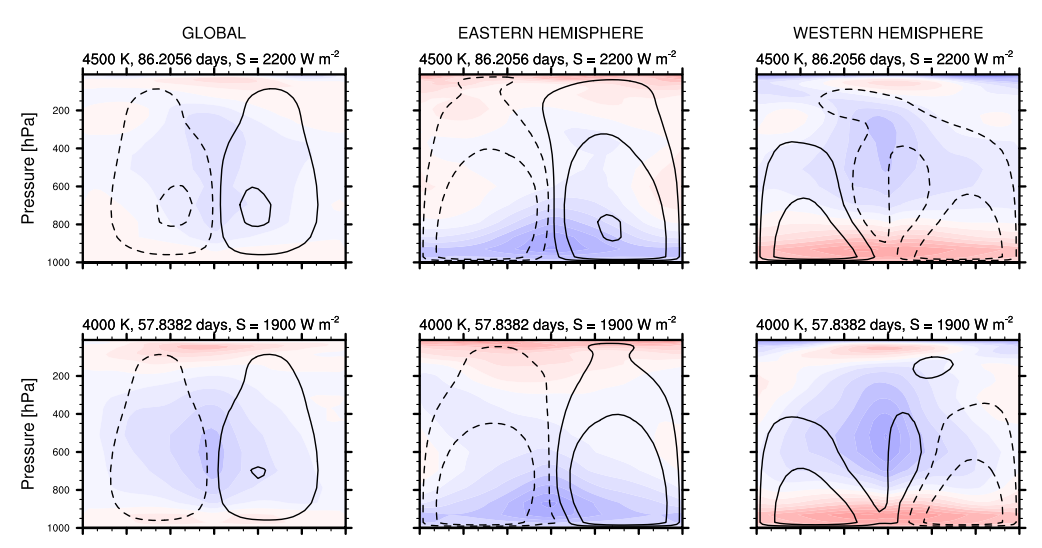

$\mathrm{m} \mathrm{s}^{-1}$
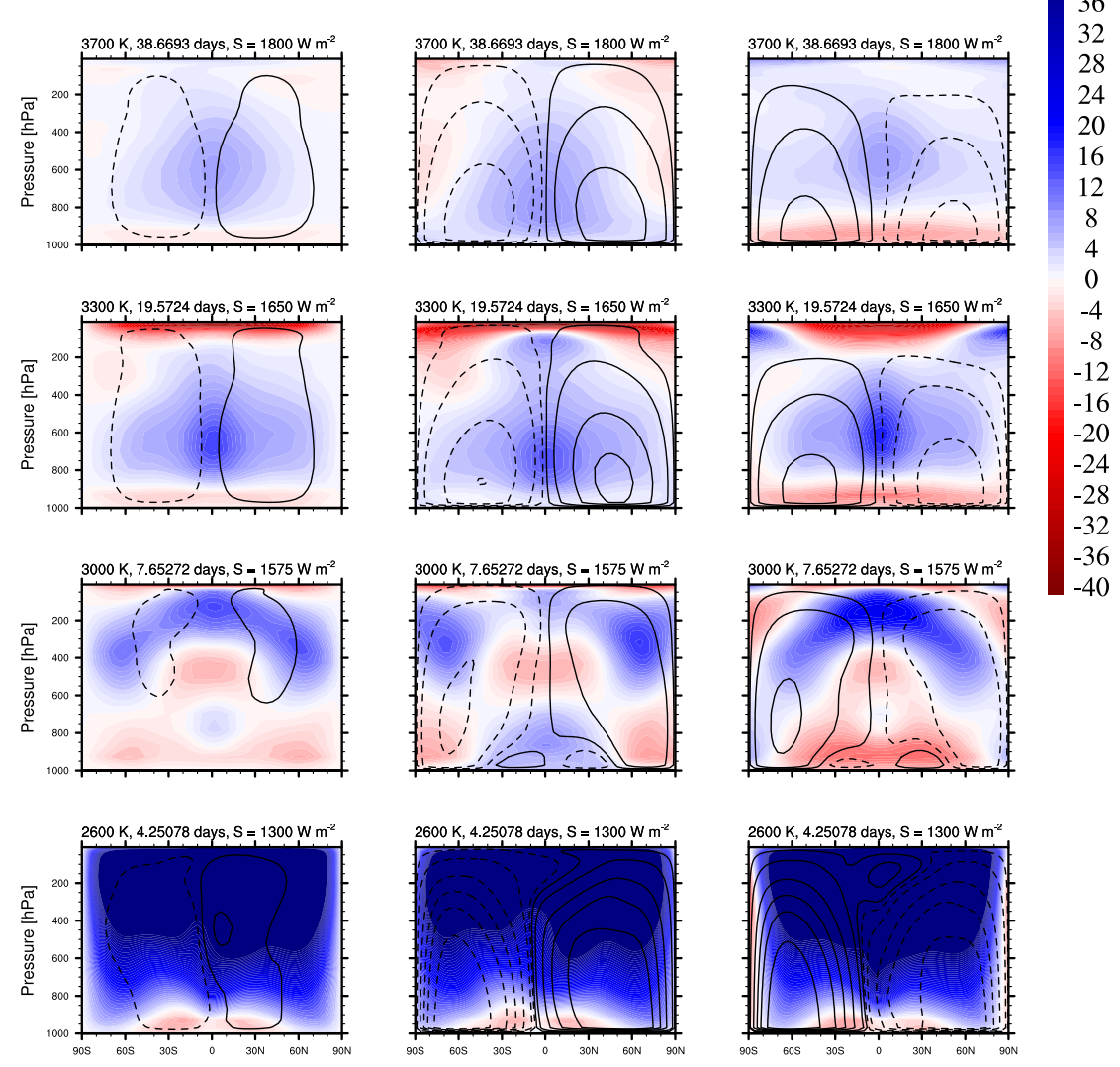

Fig. 9. - The mean meridional circulation (line contours) and zonal mean zonal wind (shading) are averaged across the entire planet (first column), eastern hemisphere (second column), and western hemisphere (third column) from the sub-stellar point. Note that all simulations show circulation patterns in the opposite direction when comparing hemispheres. Contours are drawn at an interval of $\pm\{20,100,300,500,700,1000\} \times 10^{9} \mathrm{~kg} \mathrm{~s}^{-1}$. Solid contours indicate clockwise circulation, and dashed contours indicate counter-clockwise circulation. 


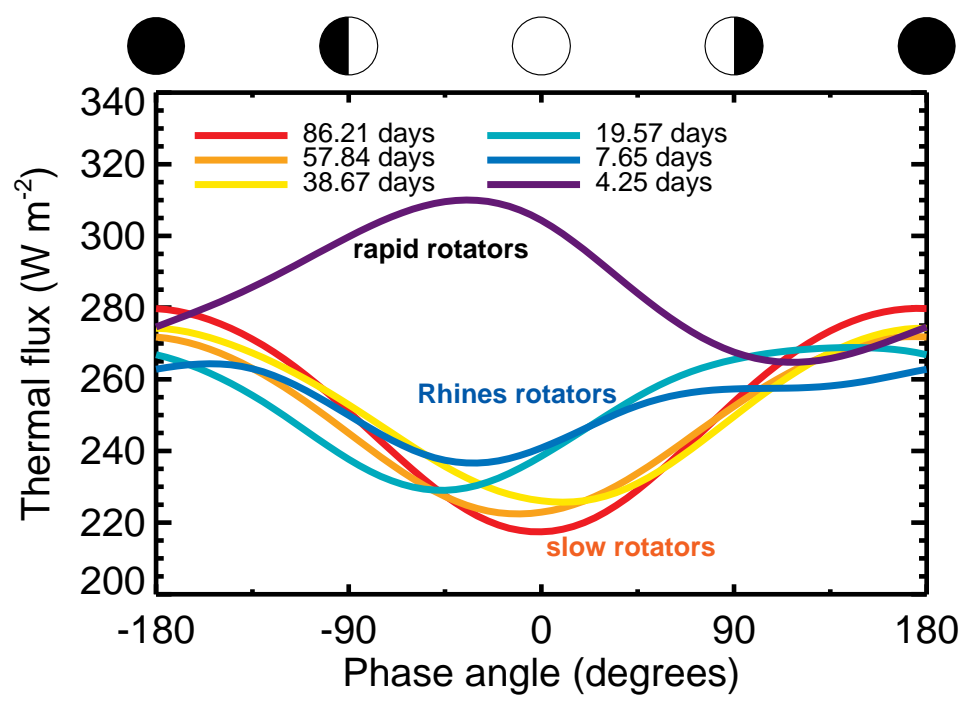

Fig. 10.- Thermal phase curves can identify synchronously rotating habitable zone planets as slow rotators (minimum thermal flux near $0^{\circ}$ ), Rhines rotators (minimum thermal flux near $-45^{\circ}$ ), and rapid rotators (maximum thermal flux near $-45^{\circ}$ ). 


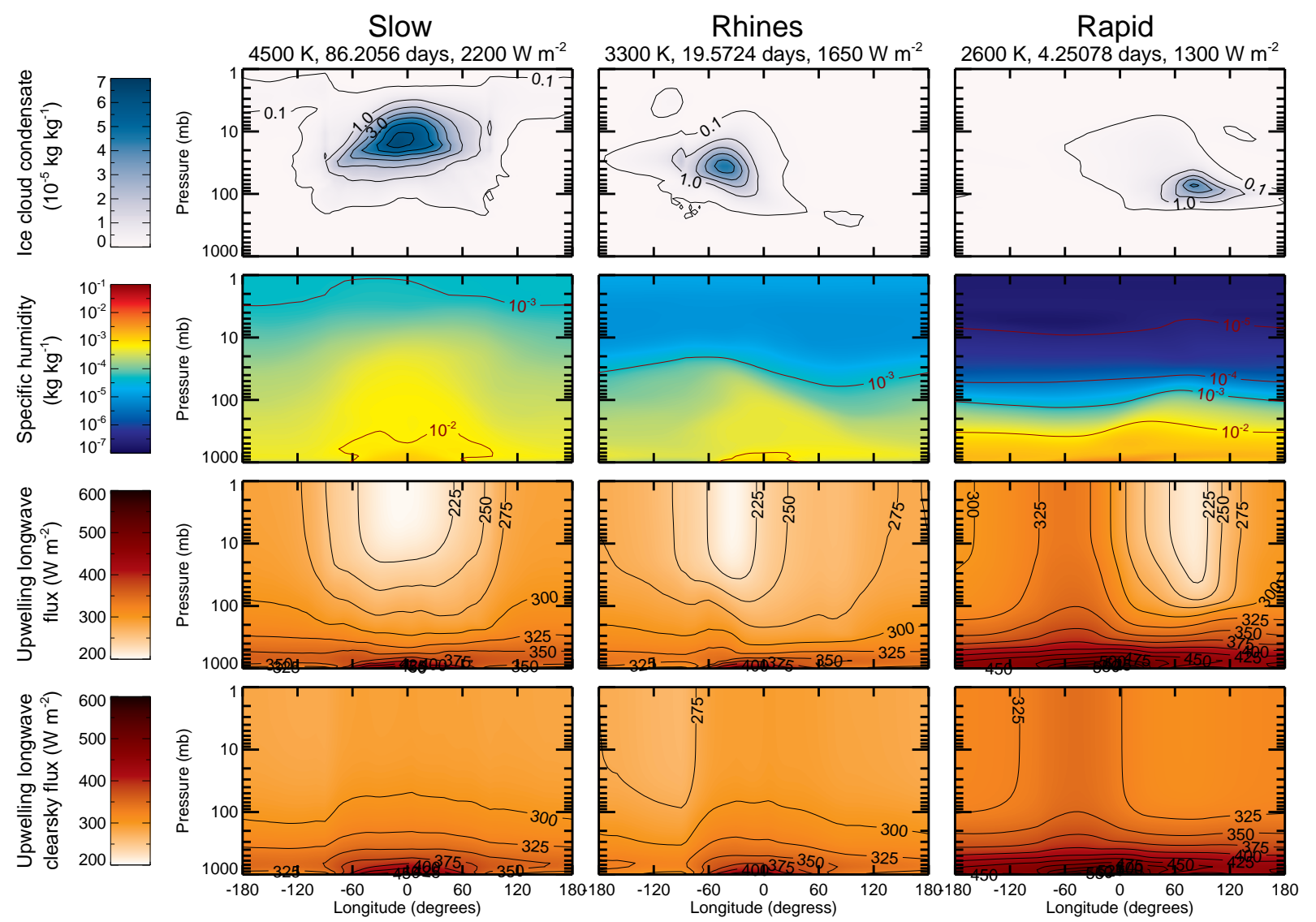

Fig. 11. - Vertical profiles taken along the equator for ice cloud condensate mass mixing ratio (top row), specific humidity (second row), upwelling longwave flux (third row), and upwelling longwave clearsky flux (bottom row). The substellar point is located at $0^{\circ}$ longitude. Ice clouds form in regions of high specific humidity and cold temperatures, typically above $100 \mathrm{mb}$. The minimum in upwelling longwave flux corresponds to the maximum in ice cloud condensate for all simulations, because ice clouds are efficient greenhouse absorbers. Slow rotators show areas of high specific humidity and cirrus clouds located immediately over the substellar point. Water vapor, ice clouds, and the associated minima in the outgoing longwave flux are shifted westward of the substellar point for Rhines rotators and eastward of the substellar point for rapid rotators. When clouds are ignored in the radiative transfer calculation, the upwelling longwave clearsky flux appears generally uniform across all longitudes. 